\title{
Palladium Catalyzed Suzuki Cross-Coupling of 3-Iodo-2-(methylthio)-benzo[b]furan Derivatives: Synthesis of 3-Aryl-2-(methylthio)benzo[b]furans
}

\author{
Rafaela M. Gay, Flávia Manarin, Ricardo Brandão and Gilson Zeni* \\ Departamento de Química, Centro de Ciências Naturais e Exatas, \\ Universidade Federal de Santa Maria, 97105-900 Santa Maria-RS, Brazil
}

\begin{abstract}
Neste trabalho desenvolvemos um método seletivo e eficiente para a síntese de derivados de 3-aril-2-(tiometil)-benzo[b]furanos, via reação de acoplamento com ácidos borônicos, catalisada por paládio. As condições reacionais usadas foram brandas e compatíveis com ácidos borônicos contendo substituintes retiradores, doadores de elétrons ou neutros.

A selective and efficient method for the synthesis of 3-aryl-2-(methylthio)benzo[ $b]$ furans derivatives by palladium catalyzed cross-coupling reaction with boronic acids has been developed. The reaction proceeded cleanly under mild conditions and was performed with aryl boronic acids bearing electron-withdrawing, electron donating and neutral substituents.
\end{abstract}

Keywords: palladium, Suzuki cross-coupling, benzofuran, boronic acids

\section{Introduction}

The construction of benzo[b]furans substituted in the 2,3 positions is of great synthetic interest due to the presence of this moiety in many complex compounds, which have potential therapeutic applications. ${ }^{1}$ In this context, the preparation of 2,3-disubstituted benzo[b] furans represents a synthetic challenge and the palladiumcatalyzed Suzuki coupling is one of the most straightforward methodologies to synthesize 3 -arylated-benzofurans. ${ }^{2}$ The palladium-catalyzed Suzuki cross-coupling reaction of aryl halides with organoboron reagents has become a common and convenient synthetic method in organic chemistry. ${ }^{3}$ Many examples of Suzuki coupling reaction of heterocyclic halides with boronic acids have appeared in the literature over the past two decades as a promising synthetic tool. ${ }^{4}$ More recently, significant advances have been made in the use of organoboron reagents as coupling partners in a number of palladium-mediated carbon-carbon bond formation. Among them, the use of potassium organotrifluoroborates, as the organoboron coupling partner, has some of these advantages are higher nucleophilicity, air-stability and ready access in comparison to boronic acids and boronic esters. ${ }^{5}$

*e-mail: gzeni@pq.cnpq.br
In addition, the presence of a sulfide group at the 2-position of benzo[b]furan is significant, since it could be easily oxidized to 2 -sulfonylbenzofurans, ${ }^{6}$ a class of compounds which has attracted tremendous interest from the pharmaceutical industry. During the course of our research program aiming at structure-activity relationship studies in order to evaluate the pharmacology activity of heterocycles, ${ }^{7,8}$ we required the synthesis of benzofurans having an organochalcogen group at the 2-position and a substituted aromatic ring at the 3-position. Since the Suzuki reaction has proven to be successful for the selective arylation of heterocyles, ${ }^{2}$ it was of interest to explore this reaction to obtain 3-aryl-2-(methylthio) benzo[ $b]$ furan derivatives. To our knowledge this methodology has not been explored and we now wish to report the application of 3-iodo-2-(methylthio)benzo[b] furans 1a-c as substrates on the cross-coupling reaction with boronic acids $\mathbf{2} \mathbf{a}-\mathbf{k}$ in the presence of a palladium salt to obtain 3-aryl-2-(methylthio)benzo[b]furans 3a-u (Scheme 1).

The starting materials 1a-c were readily available by using the electrophilic cyclization protocol ${ }^{9}$ of 2-(methylthio)alkynylanisoles (Scheme 2). The treatment of 2-(methylthio)alkynylanisoles with iodine in $\mathrm{CH}_{2} \mathrm{Cl}_{2}$, at room temperature, led to the formation of $\mathbf{1 a - c}$, isolated in $87-95 \%$ yield after purification (Scheme 2). ${ }^{10}$ 
<smiles>[R]c1ccc2c(I)c(SC)oc2c1</smiles>

$\mathrm{R}=\mathrm{H}, \mathrm{Me}, \mathrm{F}$

$\mathrm{Ar}=\mathrm{C}_{6} \mathrm{H}_{5}, p-\mathrm{OMe}-\mathrm{C}_{6} \mathrm{H}_{4}, p-\mathrm{Me}_{6} \mathrm{C}_{6} \mathrm{H}_{4}, o-\mathrm{Me}-\mathrm{C}_{6} \mathrm{H}_{4}, p-\mathrm{Cl}-\mathrm{C}_{6} \mathrm{H}_{4}, p-\mathrm{Br}_{-} \mathrm{C}_{6} \mathrm{H}_{4}, p-\mathrm{Me}-\mathrm{COC}_{6} \mathrm{H}_{4}, m-\mathrm{Me}-\mathrm{COC}_{6} \mathrm{H}_{4}$, $m-\mathrm{CF}_{3}-\mathrm{C}_{6} \mathrm{H}_{4}, m-\mathrm{NO}_{2}-\mathrm{C}_{6} \mathrm{H}_{4}$, naphtyl

Scheme 1.<smiles>[R]c1ccc(OC)c(C#CSC)c1</smiles><smiles>[R]c1ccc2oc(SC)c(I)c2c1</smiles>

$$
\begin{aligned}
& R=H, 1 a-93 \% \\
& R=M e, 1 b-95 \% \\
& R=F, 1 c-87 \%
\end{aligned}
$$

Scheme 2.

\section{Results and Discussion}

Our initial studies on Suzuki cross-coupling focused on the development of an optimum set of reaction conditions. In this way, 3-iodo-2-(methylthio)benzo[b]furan 1a and phenylboronic acid $\mathbf{2 a}$ were used as standard substrates. Thus, the reaction of $\mathbf{1 a}(0.5 \mathrm{mmol})$, boronic acid $\mathbf{2 a}$ $(0.75 \mathrm{mmol})$, and a solution of $\mathrm{K}_{2} \mathrm{CO}_{3}(1.0 \mathrm{mmol})$ in $\mathrm{H}_{2} \mathrm{O}$
(0.4 mL), using DMF (4.0 mL) as solvent, at reflux with different palladium catalysts was tested (Table 1). As shown in Table 1 , both $\operatorname{Pd}(0)$ and $\operatorname{Pd}(\mathrm{II})$ catalysts with different ligands were tested. The best result was obtained using $\mathrm{Pd}\left(\mathrm{PPh}_{3}\right)_{4}(5 \mathrm{~mol} \%)$ which gave the desired product $\mathbf{3 a}$ in $97 \%$ yield (Table 1; entry 1). It is important to note that when the amount of catalyst was reduced from 5 to $2 \mathrm{~mol} \%$, a small decrease in the yield was observed (Table 1; entry 7).

Table 1. Effects of palladium catalysts on reaction of $\mathbf{1 a}$ and $\mathbf{2} \mathbf{a}^{\mathrm{a}}$

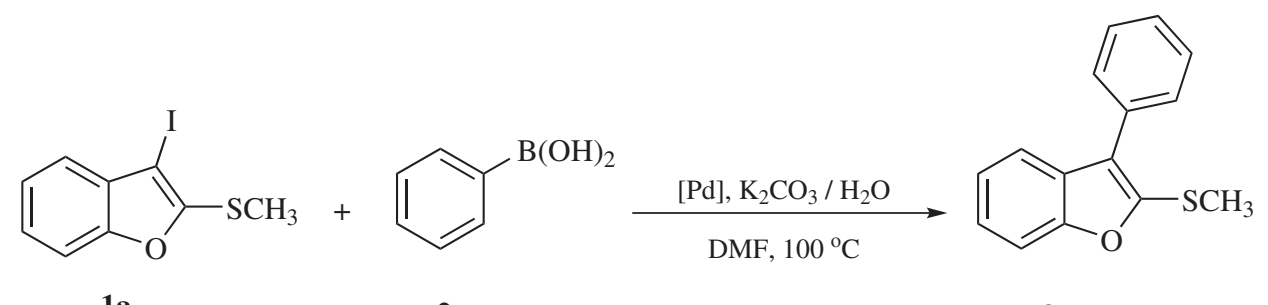

$1 \mathbf{a}$

$2 \mathbf{a}$

$3 \mathbf{a}$

\begin{tabular}{lcc}
\hline Entry & Catalysts $(\mathrm{mol} \%)$ & Yield $/(\%)^{\mathrm{b}}$ \\
\hline 1 & $\mathrm{Pd}\left(\mathrm{PPh}_{3}\right)_{4}(5)$ & 97 \\
2 & $\mathrm{Pd}(\mathrm{acac})_{2}(5)$ & traces \\
3 & $\mathrm{PdCl}_{2}\left(\mathrm{PPh}_{3}\right)_{2}(5)$ & 83 \\
4 & $\mathrm{PdCl}_{2}\left(\mathrm{PhCN}_{2}(5)\right.$ & traces \\
5 & $\mathrm{PdCl}_{2}(5)$ & $\mathrm{nr}$ \\
6 & $\mathrm{Pd}\left(\mathrm{OAc}_{2}(5)\right.$ & 15 \\
7 & $\mathrm{Pd}\left(\mathrm{PPh}_{3}\right)_{4}(2)$ & 93 \\
8 & - & $\mathrm{nr}$ \\
\hline
\end{tabular}

${ }^{a}$ Reactions were performed in the presence of $\mathbf{1 a}(0.5 \mathrm{mmol}), \mathbf{2 a}(0.75 \mathrm{mmol}), \mathrm{K}_{2} \mathrm{CO}_{3}(1.0 \mathrm{mmol}), \mathrm{H}_{2} \mathrm{O}(0.4 \mathrm{~mL})$, in DMF $(4.0 \mathrm{~mL})$, under $100{ }^{\circ} \mathrm{C}$, during $1 \mathrm{~h}$. ${ }^{\text {b}}$ Yields are given by GC analysis. $\mathrm{nr}=$ no reaction. 
Our experiments also showed that in the absence of catalyst the product was not detected, and the starting material was recovered (Table 1 ; entry 8 ).

Regarding the influence of solvent, optimal results were achieved using DMF (Table 2, entry 1). By using dioxane, $\mathrm{CH}_{3} \mathrm{CN}$ and THF (Table 2, entries 2, 4 and 6) good yields were also obtained, while the use of DME (Table 2, entry 5) did not give the desired product 3a. We also observed that the nature of the base was critical for the success of the coupling. When the reaction was carried out with an aqueous solution of inorganic bases such as $\mathrm{K}_{3} \mathrm{PO}_{4}, \mathrm{Cs}_{2} \mathrm{CO}_{3}$ and $\mathrm{Na}_{2} \mathrm{CO}_{3}$ the coupling product was obtained in good yields (Table 2; entries 7-9), while the use of $\mathrm{NaOH}$ (Table 2, entry 10) furnished the desired coupling product in low yield. It is also important to mention that when dry base were used in place of an aqueous solution, only $54 \%$ of product was obtained (Table 2; entry 11). Careful analysis of the results revealed that the optimum condition for this coupling reaction was found to be the use of $\mathrm{Pd}\left(\mathrm{PPh}_{3}\right)_{4}(2 \mathrm{~mol} \%)$, 3-iodo-2(methylthio)-benzo[ $b]$ furan $1 \mathbf{a}(0.5 \mathrm{mmol})$, phenylboronic acid $(0.75 \mathrm{mmol})$ in DMF $(4.0 \mathrm{~mL})$ and $\mathrm{H}_{2} \mathrm{O}(0.4 \mathrm{~mL})$ at room temperature. After that, the base $\mathrm{K}_{3} \mathrm{PO}_{4}(1.0 \mathrm{mmol})$ was added and the mixture was heated at $100^{\circ} \mathrm{C}$ for $1 \mathrm{~h}$. Using this reaction condition we were able to prepare 3-aryl-2-(methylthio)benzo[b]furan derivative 3a in excellent yield. ${ }^{12}$
In order to demonstrate the efficiency of this protocol, we explored the generality of our method extending the conditions to other aryl boronic acids and 3-iodo-2(methylthio)-benzo[ $b]$ furan derivatives and the results are summarized in Table 3. Inspection of Table 3 shows that the reaction worked well for a variety of arylboronic acids. A closer inspection of the results revealed that the reaction is sensitive to the electronic effect of the aromatic ring attached in the arylboronic acid. For example, arylboronic acids bearing an electron-donating group, methyl or methoxyl, at the para position gave a better yield than the arylboronic acid bearing an electron-withdrawing group, halide and acetyl (Table 3; entries 2, 3 versus 4-6). These results are in full agreement with the fact that electron donating groups increase the nucleophilicity of boronic acids. We have found that steric effects had a little influence on the coupling reaction since boronic acids containing 2-methylphenyl or naphtyl group, gave similar yields than arylboronic acids with no substituent, however an increase in the reaction time was required (Table 3; entries 10, 15, 18 and 21). Differentiation in the reactivity between halogen and boron atoms can be seen by coupling showed in the experiments described in Table 1 (enties 4 and 5), which provide only the Suzuki product, without any homo-coupling product. To the best

Table 2. Study of base and solvent effects on cross-coupling reaction ${ }^{\mathrm{a}}$

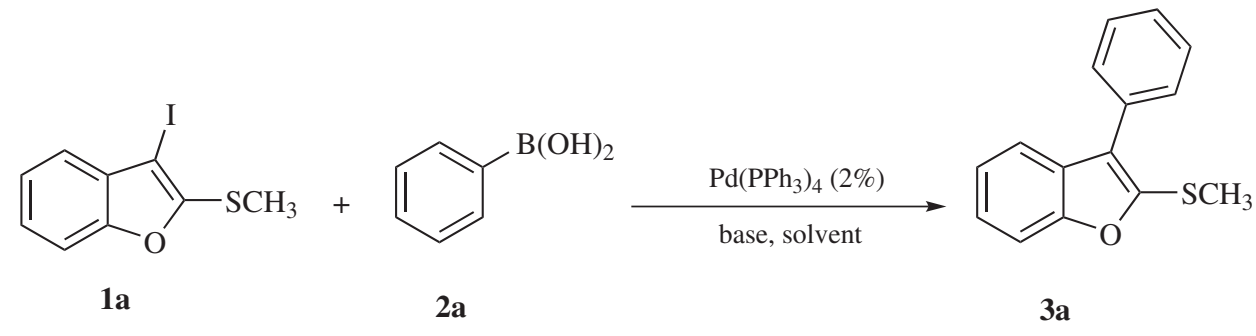

\begin{tabular}{lccc}
\hline Entry & Base & Solvent & Yield $\mathbf{3 a} /(\%)^{\mathrm{b}}$ \\
\hline 1 & $\mathrm{~K}_{2} \mathrm{CO}_{3}$ & DMF & 93 \\
2 & $\mathrm{~K}_{2} \mathrm{CO}_{3}$ & Dioxane & 91 \\
3 & $\mathrm{~K}_{2} \mathrm{CO}_{3}$ & Toluene & 45 \\
4 & $\mathrm{~K}_{2} \mathrm{CO}_{3}$ & $\mathrm{CH}$ & 90 \\
5 & $\mathrm{~K}_{2} \mathrm{CO}_{3}$ & $\mathrm{DME}$ & $\mathrm{nr}$ \\
6 & $\mathrm{~K}_{2} \mathrm{CO}_{3}$ & $\mathrm{THF}$ & 70 \\
7 & $\mathrm{~K}_{3} \mathrm{PO}_{4}$ & $\mathrm{DMF}$ & 64 \\
8 & $\mathrm{Na}_{2} \mathrm{CO}_{3}$ & $\mathrm{DMF}$ & 60 \\
9 & $\mathrm{Cs}_{2} \mathrm{CO}_{3}$ & $\mathrm{DMF}$ & 73 \\
10 & $\mathrm{NaOH}_{11}$ & $\mathrm{DMF}$ & 36 \\
11 & $\mathrm{~K}_{2} \mathrm{CO}_{3}$ & $\mathrm{DMF}$ & $54^{c}$ \\
\hline
\end{tabular}

${ }^{a}$ Reactions were performed by using $\mathbf{1 a}(0.5 \mathrm{mmol}), \mathbf{2 a}(0.75 \mathrm{mmol}), \mathrm{Pd}\left(\mathrm{PPh}_{3}\right)_{4}(2 \mathrm{~mol} \%)$, base $(1.0 \mathrm{mmol}), \mathrm{H}_{2} \mathrm{O}(0.4 \mathrm{~mL})$ with different solvents $(4.0 \mathrm{~mL})$, under $100{ }^{\circ} \mathrm{C}$. ${ }^{b}$ Yields are given by GC analysis. ${ }^{\circ}$ This reaction was carried out using dry base. 
Table 3. Coupling products obtained using 3-iodo-2(methylthio)benzo[ $b]$ furans $\mathbf{1 a - c}$ and boronic acids $\mathbf{2} \mathbf{a}^{-\mathbf{k}^{\mathrm{a}}}$<smiles></smiles><smiles>[R]c1ccc2c([Al])c(C)oc2c1</smiles>

$\mathrm{R}=\mathrm{H}, \mathrm{Me}, \mathrm{F}$

Entry Substrate

7

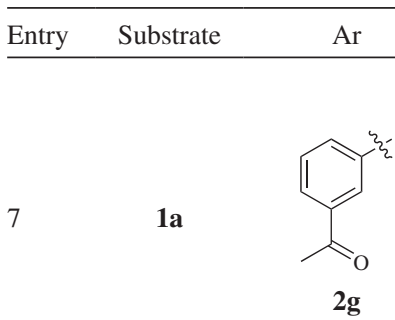

8

9

10

$1 \mathrm{a}$<smiles>FC(F)(F)c1cccc(I)c1</smiles>

$1 a$

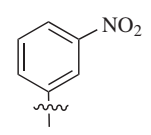

$2 \mathrm{~h}$
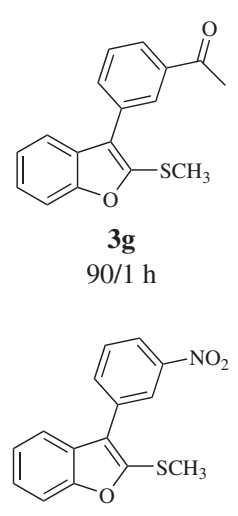

$3 \mathbf{h}$

$57 / 12 \mathrm{~h}$

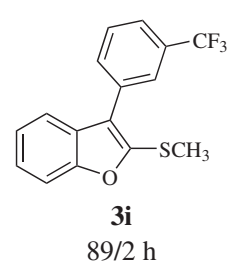

$\overbrace{2 j}^{2 i n}$

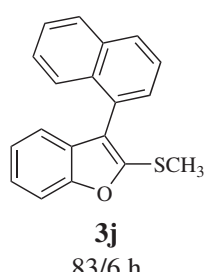

11

1b

2a

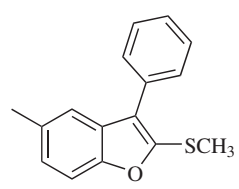

$3 \mathbf{k}$

95/1 h<smiles>CSc1oc2ccc(C)cc2c1-c1ccc(Cl)cc1</smiles>

31

$86 / 2$ h 
Table 3. continuation

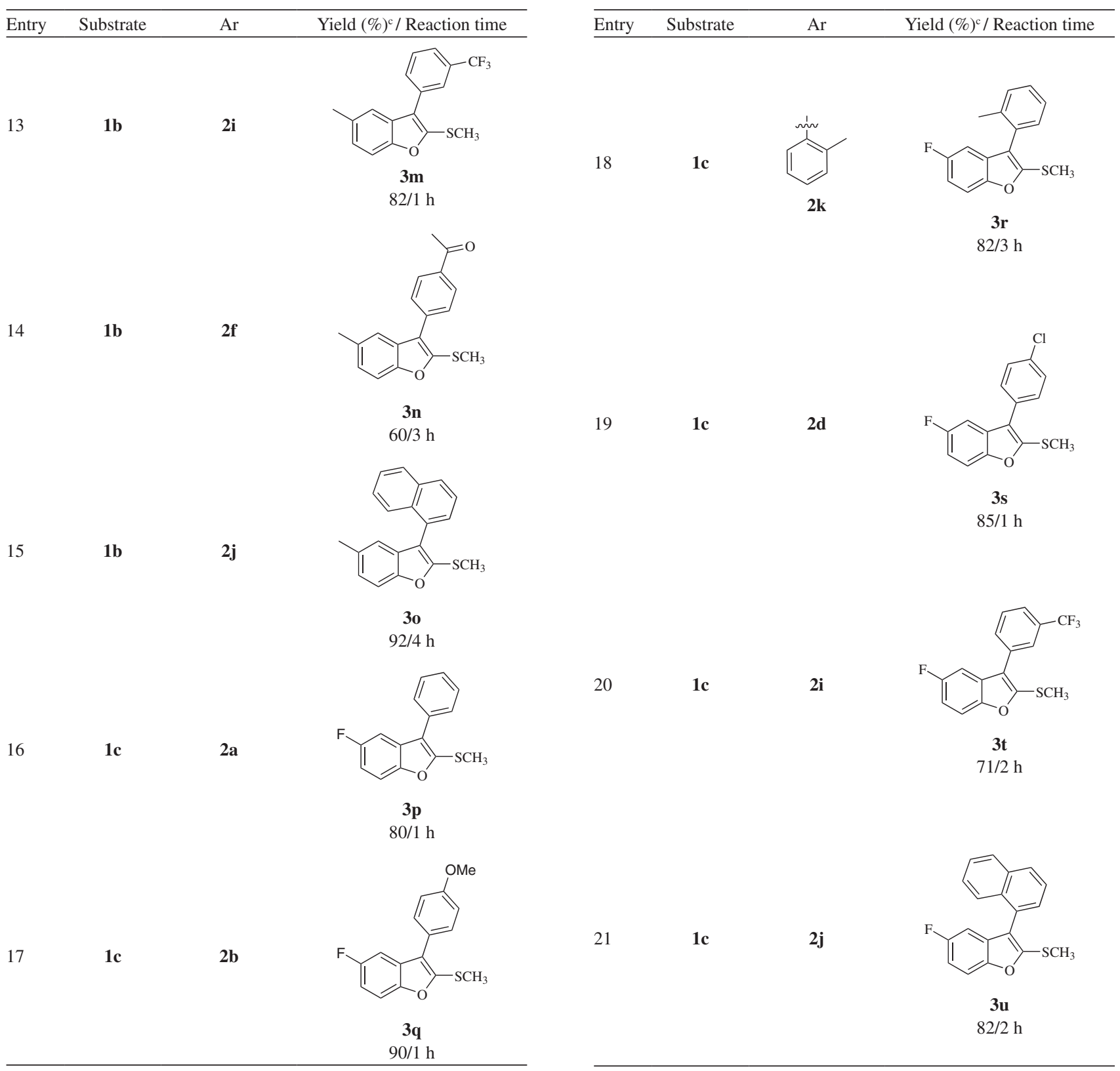

${ }^{a}$ Reactions were performed by using 1a-c $(0.5 \mathrm{mmol}), \mathbf{2 a - k}(0.75 \mathrm{mmol}), \mathrm{Pd}\left(\mathrm{PPh}_{3}\right)_{4}(2 \mathrm{~mol} \%), \mathrm{K}_{2} \mathrm{CO}_{3}(1.0 \mathrm{mmol}), \mathrm{H}_{2} \mathrm{O}(0.4 \mathrm{~mL})$ in DMF $(4.0 \mathrm{~mL}), \mathrm{under}$ $100{ }^{\circ} \mathrm{C} .{ }^{\mathrm{b}}$ Yield given by GC analysis. ${ }^{\mathrm{c} Y i e l d s}$ given for isolated products.

of our knowledge, aryl halogen could react with boronic acids in the presence of palladium catalysts to afford biaryl products. ${ }^{11}$ In the example reported in this study, the halogen substituent was not affected. In an attempt to broaden the scope of our methodology, the possibility of performing the reaction with other 3-iodo-2-(methylthio)benzofurans was also investigated. Then substrates $\mathbf{1 b}$ and 1c, which have methyl and fluoro group in the side chain, were efficiently cross-coupled. Under these conditions, both electron-rich and electron-poor arylboronic acids produced the desired products in good yields (Table 3; entries 11-21).

Alternatively, under the same conditions described to boronic acids, we were able to use other organoboron source, the aryltrifluoroborates (Scheme 3). ${ }^{5}$ In this way, 3-iodo-2-(methylthio)-benzofuran 1a underwent palladium cross-coupling with aryltrifluoroborates furnishing the corresponding 3-aryl-2-(methylthio)benzo[b]furan in good yields. 
<smiles>CSc1oc2ccccc2c1I</smiles>

1a

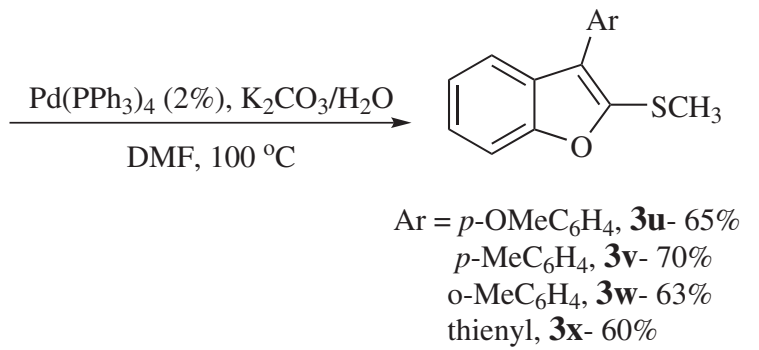

Scheme 3.

\section{Conclusions}

In summary, we have explored the Suzuki cross-coupling reaction of arylboronic acids with 3-iodo-2-(methylthio)benzofurans using a catalytic amount of $\mathrm{Pd}\left(\mathrm{PPh}_{3}\right)_{4}$. In addition, the same procedure used to aryl boronic acids was efficiently extended to aryltrifluoroborates. The reaction proceeded cleanly under mild reaction conditions, short reaction time and was performed with aryl boronic acids bearing electron withdrawing, electron donating, and neutral substituents. It is important to point out that this route permits an easy and efficient access to highly substituted benzofurans.

\section{Supplementary Information}

Supplementary data (Experimental details and caracterization of the compounds) are available free of charge at http://jbcs.sbq.org.br, as PDF file.

\section{Acknowledgments}

We are grateful to Conselho Nacional de Desenvolvimento Científico e Tecnológico (CNPq), Coordenação de Aperfeiçoamento de Pessoal de Nível Superior (CAPES) and Fundação de Amparo à Pesquisa do Estado do Rio Grande do Sul (FAPERGS), for the fellowship and financial support.

\section{References}

1. Kirilmis, C.; Ahmedzade, M.; Servi, S.; Koca, M.; Kizirgil, A.; Kazaz, C.; Eur. J. Med. Chem. 2008, 43, 300; Schultz, D. M.; Prescher, J. A.; Kidd, S.; Marona-Lewicka, D.; Nichols, D. E.; Monte, A.; Bioorg. Med. Chem. 2008, 16, 6242; Ando, K.; Kawamura, Y.; Akai, Y.; Kunitomo, J.; Yokomizo, T.; Yamashita, M.; Ohta, S.; Ohishi, T.; Ohishi, Y.; Org. Biomol. Chem. 2008, 6, 296; Sakata, Y.; Kuramoto, M.; Ando, K.; Yamaguchi, M.; Kawasaki, I.; Kunitomo, J-I.; Yokomizo, T.; Ohishi, Y.; Org. Biomol. Chem. 2007, 5, 3083.

2. Tung, D. T.; Tuan, D. T.; Rasool, N.; Villinger, A.; Reinke, H.; Fischer, C.; Langer, P.; Adv. Synth. Catal. 2009, 351, 1595;
Ullah, I.; Khera, R. A.; Hussain, M.; Villinger, A.; Langer, P.; Tetrahedron Lett. 2009, 50, 4651; Dang, T. T.; Rasool, N.; Dang, T. T.; Reinke, H.; Langer, P.; Tetrahedron Lett. 2007, 48, 845; Tuan, D. T.; Tung, D. T.; Langer, P.; Synlett 2006, 2812; Bellur, E.; Langer, P.; Eur. J. Org. Chem. 2005, 22, 4815.

3. Miyaura, N.; Suzuki, A.; Chem. Rev. 1995, 95, 2457; Suzuki, A.; Pure Appl. Chem. 1994, 66, 213; Suzuki, A. In Metal-Catalyzed Cross-Coupling Reactions; Diederich, F.; Stang, P. J., eds.; Wiley-VHC: Weinheim, 1998; Lloyd-Williams, P.; Giralt, E.; Chem. Soc. Rev. 2001, 30, 145.

4. Barancelli, D. A.; Alves, D.; Prediger, P.; Stangherlin, E. C.; Nogueira, C. W.; Zeni, G.; Synlett 2008, 119; Stanforth, S. P.; Tetrahedron 1998, 54, 263; Cailly, T.; Fabis, F.; Legay, R.; Oulyadi, H.; Rault, S.; Tetrahedron 2007, 63, 71; Sicre, C.; Alonso-Gomez, J. L.; Cid, M. M.; Tetrahedron 2006, 62, 11063; Schnurch, M.; Flasik, R.; Khan, A. F.; Spina, M.; Mihovilovic, M. D.; Stanetty, P.; Eur. J. Org. Chem. 2006, 15, 3283.

5. Molander, G. A.; Ellis, N.; Acc. Chem. Res. 2007, 40, 275; Molander, G. A.; Petrillo, D. E.; Landzberg, N. R.; Rohanna, J. C.; Biolatto, B.; Synlett 2005, 1763; Molander, G. A.; Figueroa, R.; Aldrichimica Acta 2005, 38, 49; Molander, G. A.; Felix, L. A.; J. Org. Chem. 2005, 70, 3950; Molander, G. A.; Yun, C. S.; Ribagorda, M.; Biolatto, B.; J. Org. Chem. 2003, 68, 5534; Molander, G. A.; Biolatto, B.; J. Org. Chem. 2003, 68, 4302; Molander, G. A.; Katona, B. W.; Machrouhi, F.; J. Org. Chem. 2002, 67, 8416; Molander, G. A.; Reviro, M. R.; Org. Lett. 2002, 4, 107.

6. Kakarla, R.; Dulina, R. G.; Hatzenbuhler, N. T.; Hui, Y. W.; Sofia, M. J.; J. Org. Chem. 1996, 61, 8347.

7. Stein, A. L.; Alves, D.; Rocha, J. T.; Nogueira, C. W.; Zeni, G.; Org. Lett. 2008, 10, 4983; Alves, D.; Luchese, C.; Nogueira, C. W.; Zeni, G.; J. Org. Chem. 2007, 72, 6726; Prediger, P.; Moro, A. V.; Nogueira, C. W.; Savegnago, L.; Rocha, J. B. T.; Zeni, G.; J. Org. Chem. 2006, 71, 3786; Moro, A. V.; Nogueira, C. W.; Barbosa, N. B. V.; Menezes, P. H.; Rocha, J. B. T.; Zeni, G.; J. Org. Chem. 2005, 70, 5257; Zeni, G.; Stracke, M. P.; Nogueira, C. W.; Braga, A. L.; Menezes, P. H.; Stefani, H. A.; Org. Lett. 2004, 6, 1135; Zeni, G.; Barros, O. S. D.; Moro, A. V.; Braga, A. L.; Peppe, C.; Chem. Commun. 2003, 1258; Barros, O. S. D.; Lang, E. S.; Peppe, C.; Zeni, G.; Synlett 2003, 1725 . 
8. Zeni, G.; Ludtke, D. S.; Panatieri, R. B.; Braga, A. L.; Chem. Rev. 2006, 106, 1032; Zeni, G.; Braga, A. L.; Stefani, H. A.; Acc. Chem. Res. 2003, 36, 731; Zeni, G.; Panatieri, R. B.; Lissner, E.; Menezes, P. H.; Braga, A. L.; Stefani, H. A.; Org. Lett. 2001, 3, 819; Alves, D.; Nogueira, C. W.; Zeni, G.; Tetrahedron Lett. 2005, 46, 8761.

9. Yue, D.; Yao, T.; Larock, R. C.; J. Org. Chem. 2006, 71, 62; Yue, D.; Larock, R. C.; J. Org. Chem. 2002, 67, 1905; Kesharwani, T.; Worlikar, S. A.; Larock, R. C.; J. Org. Chem. 2006, 71, 2307.

10. Manarin, F. G.; Roehrs, J. A.; Gay, R. M.; Brandao, R.; Menezes, P. H.; Nogueira, C. W.; Zeni, G.; J. Org. Chem. 2009, 74, 2153.

11. Arvela, R. K.; Leadbeater, N. E.; Org. Lett. 2005, 7, 2101; LeBlond, C. R.; Andrews, A. T.; Sowa, J. R.; Sun, Y.; Org. Lett. 2001, 3, 1555.

12. General procedure for the cross-coupling reaction: To a solution of appropriate 3-iodo-2-(methylthio)Benzo[b]furan $(0.5 \mathrm{mmol})$ in DMF $(4.0 \mathrm{~mL})$ and $\mathrm{H}_{2} \mathrm{O}(0.4 \mathrm{~mL})$ was added to $\mathrm{Pd}\left(\mathrm{PPh}_{3}\right)_{4}$ $(0.011 \mathrm{~g}, 2 \mathrm{~mol} \%)$ and $\mathrm{K}_{2} \mathrm{CO}_{3}(1 \mathrm{mmol})$, under argon. After this time, boronic acid $(0.75 \mathrm{mmol})$ was added. The mixture was then heated at $100{ }^{\circ} \mathrm{C}$ during $1-4 \mathrm{~h}$, cooled to room temperature, diluted with dichloromethane $(20 \mathrm{~mL})$, and washed with brine $(2 \times 20 \mathrm{~mL})$. The organic phase was separated, dried over $\mathrm{MgSO}_{4}$, and concentrated under vacuum. The residue was purified by flash chromatography on silica gel using hexane/ ethyl acetate as eluent. Selected spectral and analytical data for 2-(methylthio)-3-phenylBenzo[b]furan 3a: Yield: $0.111 \mathrm{~g}$ (93\%). ${ }^{1} \mathrm{H}$ NMR: $\mathrm{CDCl}_{3}, 400 \mathrm{MHz}, \delta$ (ppm): 7.61-7.59 (m, 3H), 7.50-7.46 (m, 3H), 7.37 (t, J 7.3 Hz, 1H), 7.29 (t, J 7.3 Hz, 1H), 7.23 (dd, $J 7.5 \mathrm{~Hz}$ and $J 2.5 \mathrm{~Hz}, 1 \mathrm{H}), 2.52(\mathrm{~s}, 3 \mathrm{H}) .{ }^{13} \mathrm{C} \mathrm{NMR}$ : $\mathrm{CDCl}_{3}, 100 \mathrm{MHz}, \delta$ (ppm): 155.9, 147.5, 131.8, 129.1, 128.5, 128.3, 127.4, 124.4, 122.9, 122.5, 119.7, 110.8, 16.9. MS (EI, $70 \mathrm{eV}$ ) $\mathrm{m} / z$ (relative intensity): 238 (26), 222 (17), 194 (100), 163 (23), 151 (10), 76 (8). Anal. (\%) Calc. for $\mathrm{C}_{15} \mathrm{H}_{12}$ OS: C 74.97, H 5.03. Found: C 75.15, H 5.39.

Submitted: February 4, 2010 Published online: May 11, 2010 


\title{
Palladium Catalyzed Suzuki Cross-Coupling of 3-Iodo-2-(methylthio)-benzo[b]furan Derivatives: Synthesis of 3-Aryl-2-(methylthio)benzo[b]furans
}

\author{
Rafaela M. Gay, Flávia Manarin, Ricardo Brandão and Gilson Zeni* \\ Departamento de Química, Centro de Ciências Naturais e Exatas, \\ Universidade Federal de Santa Maria, 97105-900 Santa Maria-RS, Brazil
}

\section{Experimental}

Proton nuclear magnetic resonance spectra $\left({ }^{1} \mathrm{H} \mathrm{NMR}\right)$ were obtained at $400 \mathrm{MHz}$. Spectra were recorded in $\mathrm{CDCl}_{3}$ solutions. Chemical shifts are reported in ppm, referenced to the solvent peak of $\mathrm{CDCl}_{3}$ or tetramethylsilane (TMS) as the external reference. Data are reported as follows: chemical shift $(\delta)$, multiplicity, coupling constant $(J)$ in Hertz and integrated intensity. Carbon-13 nuclear magnetic resonance spectra $\left({ }^{13} \mathrm{C} \mathrm{NMR}\right)$ were obtained at $100 \mathrm{MHz}$. Spectra were recorded in $\mathrm{CDCl}_{3}$ solutions. Chemical shifts are reported in ppm, referenced to the solvent peak of $\mathrm{CDCl}_{3}$. Abbreviations to denote the multiplicity of a particular signal are s (singlet), d (doublet), t (triplet), q (quartet), quint (quintet), sex (sextet) and m (multiplet). High resolution mass spectra were recorded on a double focusing magnetic sector mass spectrometer using EI at $70 \mathrm{eV}$. Column chromatography was performed using silica gel (230-400 mesh) following the methods described by Still (Still, W.C.; Kahn, M.; Mitra, A.; J. Org. Chem. 1978, 43, 2923). Thin layer chromatography (TLC) was performed using silica gel $\mathrm{GF}_{254}, 0.25 \mathrm{~mm}$ thickness. For visualization, TLC plates were either placed under ultraviolet light, or stained with iodine vapor, or acidic vanillin. The following solvents were dried and purified by distillation from the reagents indicated: tetrahydrofuran from sodium with a benzophenone ketyl indicator. All other solvents were ACS or HPLC grade unless otherwise noted. Air- and moisture-sensitive reactions were conducted in flame-dried or oven dried glassware equipped with tightly fitted rubber septa and under a positive atmosphere of dry nitrogen or argon. Reagents and solvents were handled using standard syringe techniques. Temperatures above room temperature were maintained by use of a mineral

*e-mail: gzeni@pq.cnpq.br oil bath with an electrically heated coil connected to controller.

\section{General procedure for the Suzuki coupling reaction}

To a solution of appropriate 3-iodo-2-(methylthio) benzo[b]furan $(0.5 \mathrm{mmol})$ in DMF $(4.0 \mathrm{~mL})$ and $\mathrm{H}_{2} \mathrm{O}$ $(0.4 \mathrm{~mL})$ was added to $\mathrm{Pd}\left(\mathrm{PPh}_{3}\right)_{4}(0.011 \mathrm{~g}, 2 \mathrm{~mol} \%)$ and $\mathrm{K}_{2} \mathrm{CO}_{3}(1 \mathrm{mmol})$, under argon. After this time, boronic acid $(0.75 \mathrm{mmol})$ was added. The mixture was then heated at $100{ }^{\circ} \mathrm{C}$ during $1-4 \mathrm{~h}$, cooled to room temperature, diluted with dichloromethane $(20 \mathrm{~mL})$, and washed with brine $(2 \times 20 \mathrm{~mL})$. The organic phase was separated, dried over $\mathrm{MgSO}_{4}$, and concentrated under vacuum. The residue was purified by flash chromatography on silica gel using hexane/ ethyl acetate as eluent.

\section{2-(Methylthio)-3-phenylbenzofuran (3a)}

Yield: $0.111 \mathrm{~g}(93 \%) .{ }^{1} \mathrm{H}$ NMR: $\mathrm{CDCl}_{3}, 400 \mathrm{MHz}, \delta$ (ppm): 7.61-7.59 (m, 3H), 7.50-7.46 (m, 3H), 7.37 (t, $J$ $7.3 \mathrm{~Hz}, 1 \mathrm{H}), 7.29$ (t, $J 7.3 \mathrm{~Hz}, 1 \mathrm{H}), 7.23$ (dd, J 7.5 Hz and $J 2.5 \mathrm{~Hz}, 1 \mathrm{H}), 2.52$ (s, 3H). ${ }^{13} \mathrm{C}$ NMR: $\mathrm{CDCl}_{3}, 100 \mathrm{MHz}$, $\delta$ (ppm): 155.9, 147.5, 131.8, 129.1, 128.5, 128.3, 127.4, 124.4, 122.9, 122.5, 119.7, 110.8, 16.9. MS (EI, $70 \mathrm{eV}) \mathrm{m} / \mathrm{z}$ (relative intensity): 238 (26), 222 (17), 194 (100), 163 (23), 151 (10), 76 (8). Anal. (\%) Calc. for $\mathrm{C}_{15} \mathrm{H}_{12} \mathrm{OS}$ : C 74.97, $\mathrm{H}$ 5.03. Found: C 75.15, H 5.39.

\section{3-(4-Methoxyphenyl)-2-(methylthio)benzofuran (3b)}

Yield: $0.108 \mathrm{~g}(80 \%)$. ${ }^{1} \mathrm{H} \mathrm{NMR}: \mathrm{CDCl}_{3}, 400 \mathrm{MHz}$, $\delta$ (ppm): 7.59 (d, J 7.6 Hz, 1H), 7.53 (d, J 8.8 Hz, 2H), 7.47 $(\mathrm{d}, J 8.0 \mathrm{~Hz}, 1 \mathrm{H}), 7.30-7.20(\mathrm{~m}, 2 \mathrm{H}), 7.03(\mathrm{~d}, J 8.8 \mathrm{~Hz}, 2 \mathrm{H})$, 3.85 (s, 3H), 2.49 (s, 3H). ${ }^{13} \mathrm{C} \mathrm{NMR:} \mathrm{CDCl}_{3}, 100 \mathrm{MHz}$, $\delta$ (ppm): 159.0, 155.4, 146.8, 130.2, 128.4, 124.4, 124.0, 122.8, 122.4, 119.7, 114.0, 110.8, 55.2, 17.1. MS (EI, $70 \mathrm{eV}) \mathrm{m} / \mathrm{z}$ (relative intensity): 269 (14), 267 (100), 252 (48), 224 (46), 181 (14), 150 (21). 
2-(Methylthio)-3-p-tolylbenzofuran (3c)

Yield: $0.116 \mathrm{~g}(92 \%) .{ }^{1} \mathrm{H}$ NMR: $\mathrm{CDCl}_{3}, 400 \mathrm{MHz}$, $\delta$ (ppm): $7.60(\mathrm{~d}, J 7.6 \mathrm{~Hz}, 1 \mathrm{H}), 7.50(\mathrm{~d}, J 7.8 \mathrm{~Hz}, 2 \mathrm{H})$, $7.44(\mathrm{~d}, J 8.0 \mathrm{~Hz}, 1 \mathrm{H}), 7.29$ (d, J 7.8 Hz, 2H), 7.25-7.17 (m, $1 \mathrm{H}), 2.50(\mathrm{~s}, 3 \mathrm{H}), 2.40(\mathrm{~s}, 3 \mathrm{H}) .{ }^{13} \mathrm{C} \mathrm{NMR}: \mathrm{CDCl}_{3}, 100 \mathrm{MHz}$, $\delta$ (ppm): 155.4, 147.1, 137.2, 129.2, 128.9, 128.7, 128.3, $124.3,122.8,122.6,119.7,110.8,21.2,16.9$. MS (EI, $70 \mathrm{eV}$ ) $\mathrm{m} / \mathrm{z}$ (relative intensity): 254 (3), 251 (100), 236 (24), 208 (58), 176 (13), 150 (19).

\section{3-(4-Bromophenyl)-2-(methylthio)benzofuran (3e)}

Yield: $0.111 \mathrm{~g}(70 \%) .{ }^{1} \mathrm{H}$ NMR: $\left(\mathrm{CDCl}_{3}, 400 \mathrm{MHz}\right)$ $\delta$ (ppm): $7.60(\mathrm{~d}, J 8.5 \mathrm{~Hz}, 2 \mathrm{H}), 7.54(\mathrm{~d}, J 7.6 \mathrm{~Hz}, 1 \mathrm{H})$, 7.47-7.44 (m, 3H), 7.31-7.21 (m, 2H), $2.51(\mathrm{~s}, 3 \mathrm{H})$. ${ }^{13} \mathrm{C}$ NMR: ( $\left.\mathrm{CDCl}_{3}, 50 \mathrm{MHz}\right), \delta(\mathrm{ppm}): 155.4,147.7,131.7$, 130.7, 130.5, 124.6, 123.1, 121.4, 121.3, 119.4, 110.9, 16.8 . MS (EI, $70 \mathrm{eV}$ ) $\mathrm{m} / \mathrm{z}$ (relative intensity): 318 (10), 221 (100), 193 (11), 161 (14), 150 (33).

1-(4-(2-(Methylthio)benzofuran-3-yl)phenyl)ethanone (3f)

Yield: $0.098 \mathrm{~g}(70 \%) .{ }^{1} \mathrm{H}$ NMR: $\left(\mathrm{CDCl}_{3}, 400 \mathrm{MHz}\right)$ $\delta$ (ppm): 8.08 (d, J 8.3 Hz, 2H), $7.70(\mathrm{~d}, J 8.3 \mathrm{~Hz}, 2 \mathrm{H}), 7.61$ $(\mathrm{d}, J 6.8 \mathrm{~Hz}, 1 \mathrm{H}), 7.50(\mathrm{~d}, J 7.6 \mathrm{~Hz}, 1 \mathrm{H}), 7.34-7.25(\mathrm{~m}, 2 \mathrm{H})$, 2.65 (s, 3H), 2.56 (s, 3H). ${ }^{13} \mathrm{C}$ NMR: $\left(\mathrm{CDCl}_{3}, 50 \mathrm{MHz}\right)$, $\delta$ (ppm): 197.5, 155.5, 148.7, 136.9, 135.8, 129.0, 128.6, 127.8, 124.6, 123.3, 121.1, 119.4, 111.0, 26.6, 16.6. MS (EI, $70 \mathrm{eV}$ ) $\mathrm{m} / z$ (relative intensity): 280 (17), 279 (100), 264 (35), 221 (12), 150 (21), 43 (58).

1-(3-(2-(Methylthio)benzofuran-3-yl)phenyl)ethanone (3g)

Yield: $0.126 \mathrm{~g}(90 \%) .{ }^{1} \mathrm{H}$ NMR: $\left(\mathrm{CDCl}_{3}, 400 \mathrm{MHz}\right)$ $\delta$ (ppm): $8.02(\mathrm{t}, J 2.0 \mathrm{~Hz}, 1 \mathrm{H}), 7.95(\mathrm{dt}, J 7.3 \mathrm{~Hz}$ and $J$ $1.2 \mathrm{~Hz}, 1 \mathrm{H}), 7.80(\mathrm{dt}, J 7.3 \mathrm{~Hz}$ and $J 1.2 \mathrm{~Hz}, 1 \mathrm{H}), 7.59$ $7.54(\mathrm{~m}, 2 \mathrm{H}), 7.49-7.47(\mathrm{~m}, 1 \mathrm{H}), 7.30(\mathrm{td}, J 7.3 \mathrm{~Hz}$ and $J$ $1.4 \mathrm{~Hz}, 1 \mathrm{H}), 7.25$ (td, $J 7.5 \mathrm{~Hz}$ and $J 1.3 \mathrm{~Hz}, 1 \mathrm{H}), 2.64$ (s, $3 \mathrm{H}), 2.53$ (s, $3 \mathrm{H}) \cdot{ }^{13} \mathrm{C}$ NMR: $\left(\mathrm{CDCl}_{3}, 50 \mathrm{MHz}\right), \delta(\mathrm{ppm})$ : 197.6, 155.4, 148.0, 137.4, 133.4, 132.3, 128.8, 128.7, 127.8, 127.1, 124.6, 123.1, 121.4, 119.3, 110.9, 26.6, 16.7 . MS (EI, $70 \mathrm{eV}$ ) $\mathrm{m} / z$ (relative intensity): 280 (17), 278 (100), 264 (7), 150 (19).

\section{2-(Methylthio)-3-(3-nitrophenyl)benzofuran (3h)}

Yield: $0.081 \mathrm{~g}(57 \%) .{ }^{1} \mathrm{H}$ NMR: $\left(\mathrm{CDCl}_{3}, 400 \mathrm{MHz}\right)$ $\delta$ (ppm): $8.48(\mathrm{t}, J 2.0 \mathrm{~Hz}, 1 \mathrm{H}), 8.20(\mathrm{dd}, J 5.8 \mathrm{~Hz}$ and $J$ $2.0 \mathrm{~Hz}, 1 \mathrm{H}), 7.94(\mathrm{~d}, J 7.6 \mathrm{~Hz}, 1 \mathrm{H}), 7.64(\mathrm{t}, J 7.8 \mathrm{~Hz}, 1 \mathrm{H})$, $7.59(\mathrm{~d}, J 8.5 \mathrm{~Hz}, 1 \mathrm{H}), 7.50(\mathrm{~d}, J 7.8 \mathrm{~Hz}, 1 \mathrm{H}), 7.35-7.24(\mathrm{~m}$, $2 \mathrm{H}), 2.57$ (s, 3H). ${ }^{13} \mathrm{C}$ NMR: $\left(\mathrm{CDCl}_{3}, 50 \mathrm{MHz}\right), \delta$ (ppm): $155.4,148.8,148.4,134.7,133.6,129.4,127.4,124.8$, $123.5,123.4,122.0,119.9,119.0,111.0,16.5$. MS (EI, $70 \mathrm{eV}) \mathrm{m} / z$ (relative intensity): 282 (100), 250 (10), 221 (43), 192 (15), 162 (18), 150 (30).
2-(Methylthio)-3-(3-(trifluoromethyl)phenyl)benzofuran (3i)

Yield: $0.137 \mathrm{~g}(89 \%) .{ }^{1} \mathrm{H}$ NMR: $\left(\mathrm{CDCl}_{3}, 400 \mathrm{MHz}\right)$ $\delta$ (ppm): 7.88 (s, 1H), 7.78 (d, J 7.3 Hz, 1H), 7.63-7.55 (m, 3H), 7.49-7.47 (m, 1H), 7.33-7.23 (m, 2H), $2.53(\mathrm{~s}$, $3 \mathrm{H}) .{ }^{13} \mathrm{C}$ NMR: $\left(\mathrm{CDCl}_{3}, 50 \mathrm{MHz}\right), \delta$ (ppm): 155.5, 148.4, $132.8,132.3,130.8$ (q, $J 32 \mathrm{~Hz}), 129.9,129.0,125.7$ (q, $J 3.7 \mathrm{~Hz}), 124.7,124.0(\mathrm{q}, J 3.6 \mathrm{~Hz}), 123.8(\mathrm{q}, J 273 \mathrm{~Hz})$, 123.3, 119.3, 111.0, 16.7. MS (EI, $70 \mathrm{eV}$ ) $\mathrm{m} / z$ (relative intensity): 305 (100), 289 (8), 262 (95), 221 (15), 150 (13).

5-Methyl-2-(methylthio)-3-(naphthalen-1-yl)benzofuran (3o)

Yield: $0.139 \mathrm{~g}(92 \%) .{ }^{1} \mathrm{H}$ NMR: $\left(\mathrm{CDCl}_{3}, 400 \mathrm{MHz}\right)$ $\delta$ (ppm): 7.89 (d, $J 8.0 \mathrm{~Hz}, 2 \mathrm{H}), 7.73(\mathrm{~d}, J 8.5 \mathrm{~Hz}, 1 \mathrm{H})$, 7.54-7.34 (m, 5H), $7.08(\mathrm{dd}, J 7.1 \mathrm{~Hz}$ and $J 1.5 \mathrm{~Hz}, 1 \mathrm{H})$, $6.90(\mathrm{~s}, 1 \mathrm{H}), 2.40$ (s, 3H), 2.28 (s, 3H). ${ }^{13} \mathrm{C}$ NMR: $\left(\mathrm{CDCl}_{3}\right.$, $50 \mathrm{MHz}), \delta$ (ppm): 153.8, 148.9, 133.7, 132.4, 132.0, $129.9,129.3,128.4,128.3,128.2,125.9,125.8,125.6$, 125.4, 121.4, 119.9, 110.3, 21.1, 16.9. MS (EI, $70 \mathrm{eV}$ ) $\mathrm{m} / \mathrm{z}$ (relative intensity): 300 (100), 285 (27), 270 (11), 258 (32), 242 (12), 223 (7).

5-Fluoro-2-(methylthio)-3-phenylbenzofuran (3p)

Yield: $0.103 \mathrm{~g}(80 \%) .{ }^{1} \mathrm{H}$ NMR: $\left(\mathrm{CDCl}_{3}, 400 \mathrm{MHz}\right)$ $\delta$ (ppm): 7.55-7.53 (m, 2H), 7.46 (t, J 7.8 Hz, 2H), 7.37-7.33 $(\mathrm{m}, 2 \mathrm{H}), 7.24(\mathrm{dd}, J 6.1 \mathrm{~Hz}$ and $J 2.5 \mathrm{~Hz}, 1 \mathrm{H}), 6.97(\mathrm{td}, J$ $6.3 \mathrm{~Hz}$ and $J 2.9 \mathrm{~Hz}, 1 \mathrm{H}), 2.50$ (s, $3 \mathrm{H}) .{ }^{13} \mathrm{C}$ NMR: $\left(\mathrm{CDCl}_{3}\right.$, $50 \mathrm{MHz}), \delta$ (ppm): 160.6, 158.2, 151.6, 149.5, 131.2, 128.7, 128.6, 127.6, 122.1, 111.8 (d, J 26.3 Hz), 111.4 (d, $J 9.5 \mathrm{~Hz}), 105.3$ (d, $J 25.6 \mathrm{~Hz}), 16.5$. MS (EI, $70 \mathrm{eV}) \mathrm{m} / \mathrm{z}$ (relative intensity): 255 (100), 239 (64), 212 (92), 192 (12), 168 (30), 150 (6).

5-Fluoro-3-(4-methoxyphenyl)-2-(methylthio)benzofuran $(3 q)$

Yield: $0.130 \mathrm{~g}(90 \%) .{ }^{1} \mathrm{H}$ NMR: $\left(\mathrm{CDCl}_{3}, 400 \mathrm{MHz}\right)$ $\delta$ (ppm): 7.50 (d, $J 8.5 \mathrm{~Hz}, 2 \mathrm{H}), 7.37$ (dd, $J 4.9 \mathrm{~Hz}$ and $J$ $4.1 \mathrm{~Hz}, 1 \mathrm{H}), 7.24(\mathrm{dd}, J 6.1 \mathrm{~Hz}$ and $J 2.7 \mathrm{~Hz}, 1 \mathrm{H}), 7.04-6.97$ $(\mathrm{m}, 3 \mathrm{H}), 3.86$ (s, 3H), 2.51 (s, 3H). ${ }^{13} \mathrm{C}$ NMR: $\left(\mathrm{CDCl}_{3}, 50\right.$ $\mathrm{MHz}), \delta(\mathrm{ppm}): 160.6,159.1,158.2,151.6,148.8,130.0$, 123.5, 122.3, 114.2, 111.9 (d, $J 24.8 \mathrm{~Hz}$ ), 111.4 (d, $J 9.5$ Hz), 105.3 (d, $J 24.8 \mathrm{~Hz}), 55.3$, 16.7. MS (EI, $70 \mathrm{eV}) \mathrm{m} / \mathrm{z}$ (relative intensity): 285 (100), 269 (73), 242 (49), 226 (14), 155.6 (17).

\section{2-(Methylthio)-3-(thiophen-2-yl)benzofuran (3x)}

Yield: $0.074 \mathrm{~g}(60 \%) .{ }^{1} \mathrm{H}$ NMR: $\mathrm{CDCl}_{3}, 400 \mathrm{MHz}$, $\delta$ (ppm): 7.82-7.80 (m, 1H), 7.46-7.44 (m, 2H), 7.37 (dd, $J 4.0 \mathrm{~Hz}$ and $J 1.2 \mathrm{~Hz}, 1 \mathrm{H}), 7.32-7.25(\mathrm{~m}, 2 \mathrm{H}), 7.16(\mathrm{dd}, J$ $4.0 \mathrm{~Hz}$ and $J 1.4 \mathrm{~Hz}, 1 \mathrm{H}), 2.56$ (s, 3H). ${ }^{13} \mathrm{C}$ NMR: $\mathrm{CDCl}_{3}$, 
$100 \mathrm{MHz}, \delta$ (ppm): 155.3, 147.6, 133.1, 127.6, 127.2, $126.0,125.0,124.6,123.2,120.0,116.4,110.8,16.8 . \mathrm{MS}$ (EI, $70 \mathrm{eV}) \mathrm{m} / \mathrm{z}$ (relative intensity): 243 (100), 228 (75),
200 (64), 169 (15), 114 (20). HRMS calc. for $\mathrm{C}_{13} \mathrm{H}_{10} \mathrm{OS}_{2}$ : 246.0173. Found: 246.0177.

\section{Selected Spectra}<smiles>Cc1oc2ccccc2c1-c1ccccc1</smiles>

$3 a$
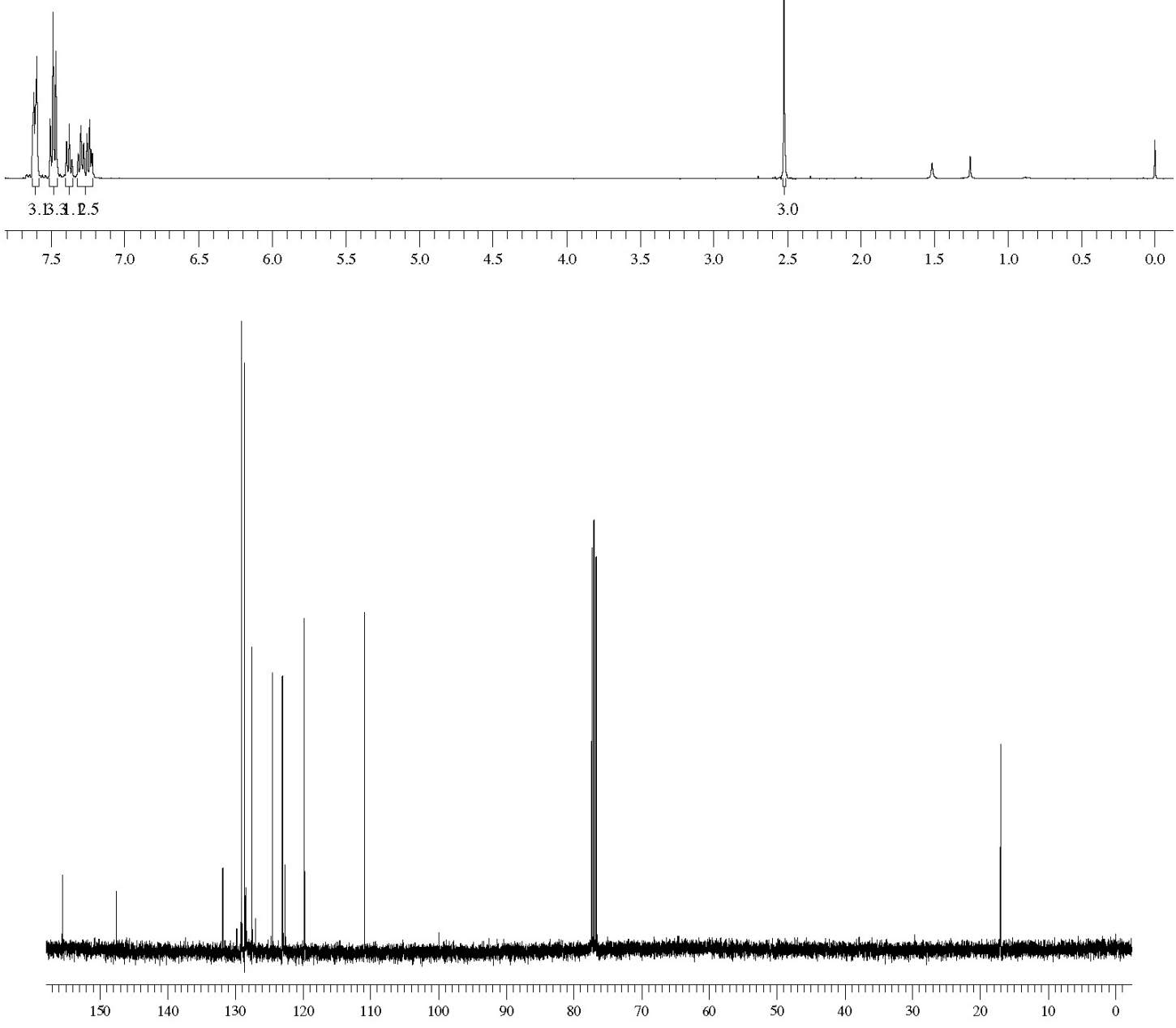

Figure S1. (up) ${ }^{1} \mathrm{H}$ NMR Spectrum (400 $\mathrm{MHz}, \mathrm{CDCl}_{3}$ ) of compound 3a; (down) ${ }^{13} \mathrm{C}$ NMR Spectrum $\left(100 \mathrm{MHz}, \mathrm{CDCl}_{3}\right.$ ) of compound $\mathbf{3 a}$. 
<smiles>COc1ccc(-c2c(C)oc3ccccc23)cc1</smiles>

3b
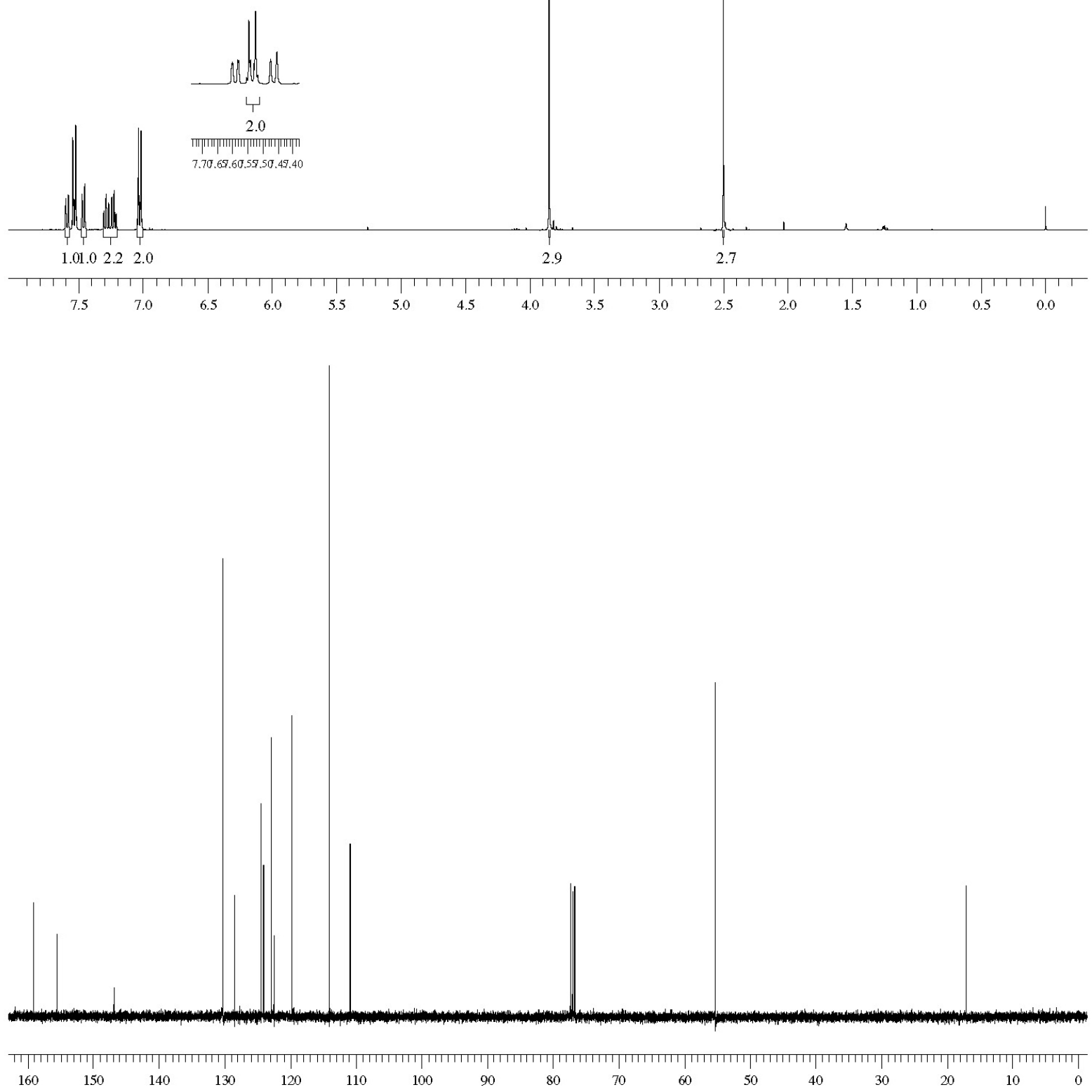

Figure S2. (up) ${ }^{1} \mathrm{H}$ NMR Spectrum (400 MHz, $\mathrm{CDCl}_{3}$ ) of compound $\mathbf{3 b}$; (down) ${ }^{13} \mathrm{C}$ NMR Spectrum (100 MHz, $\mathrm{CDCl}_{3}$ ) of compound $\mathbf{3 b}$. 


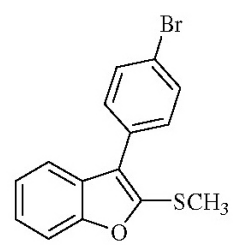

$3 \mathbf{e}$
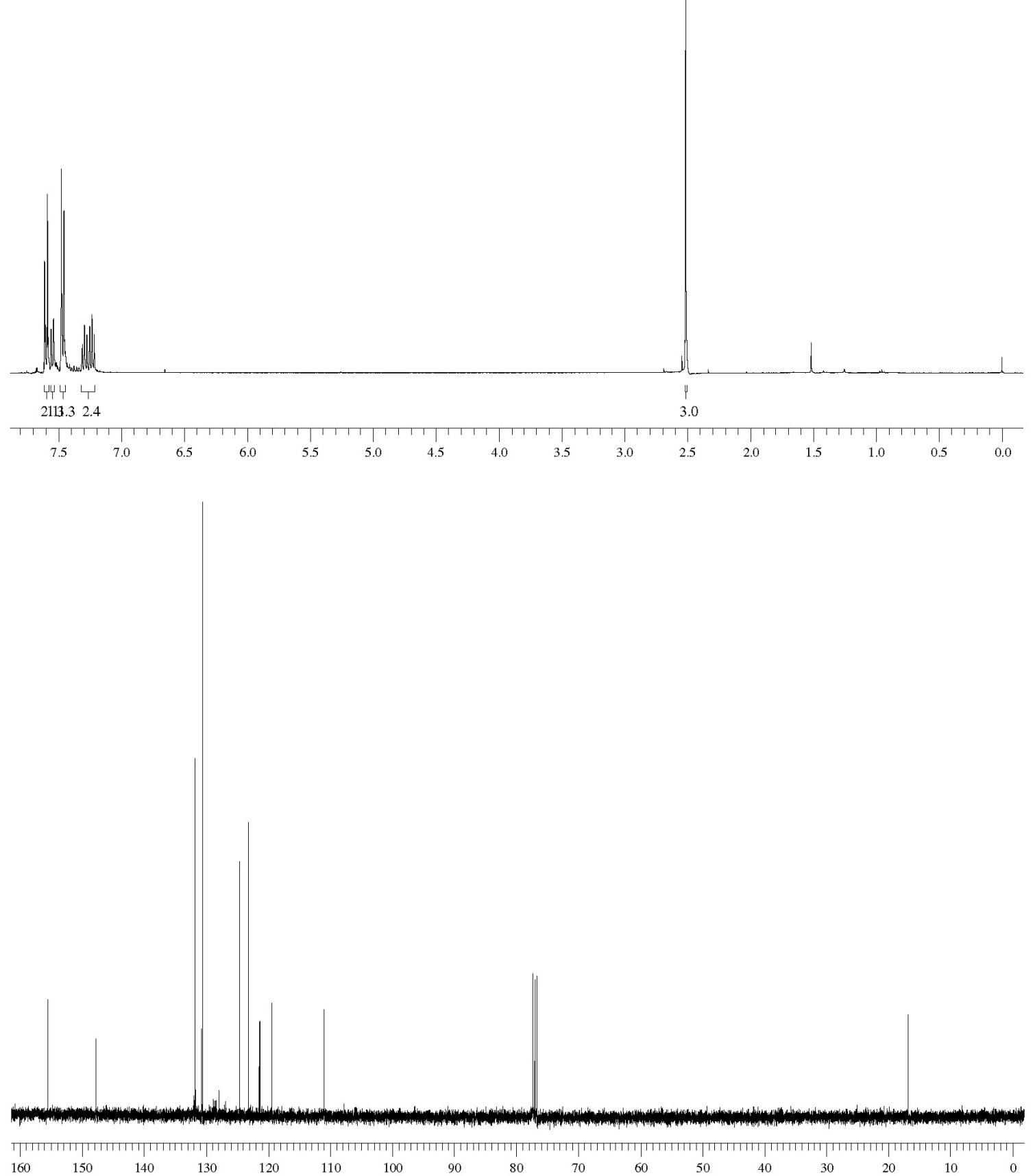

Figure S3. (up) ${ }^{1} \mathrm{H}$ NMR Spectrum (400 $\mathrm{MHz}, \mathrm{CDCl}_{3}$ ) of compound 3e; (down) ${ }^{13} \mathrm{C}$ NMR Spectrum $\left(100 \mathrm{MHz}, \mathrm{CDCl}_{3}\right.$ ) of compound $\mathbf{3 e}$. 


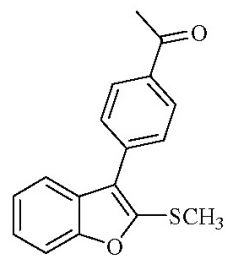

$3 \mathbf{f}$
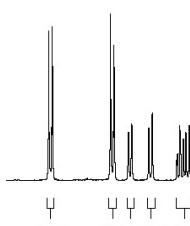

$2.1 \quad 2.1 .0 .02 .2$
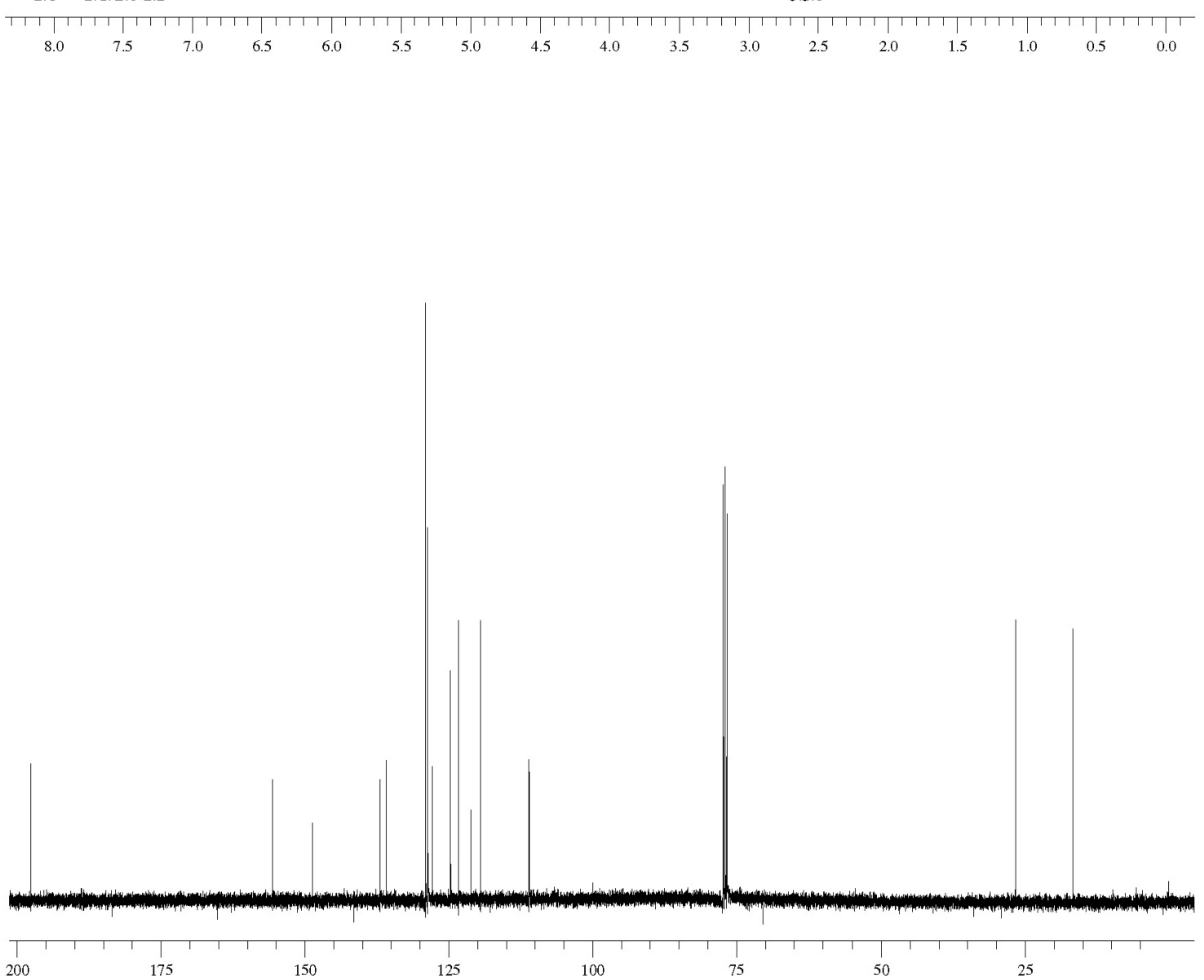

Figure S4. (up) ${ }^{1} \mathrm{H}$ NMR Spectrum (400 $\mathrm{MHz}, \mathrm{CDCl}_{3}$ ) of compound $\mathbf{3 f}$; (down) ${ }^{13} \mathrm{C}$ NMR Spectrum $\left(100 \mathrm{MHz}, \mathrm{CDCl}_{3}\right.$ ) of compound $\mathbf{3 f}$. 


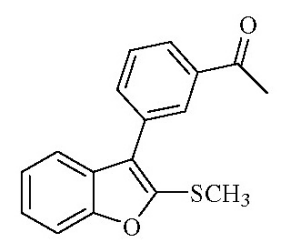

$3 \mathrm{~g}$
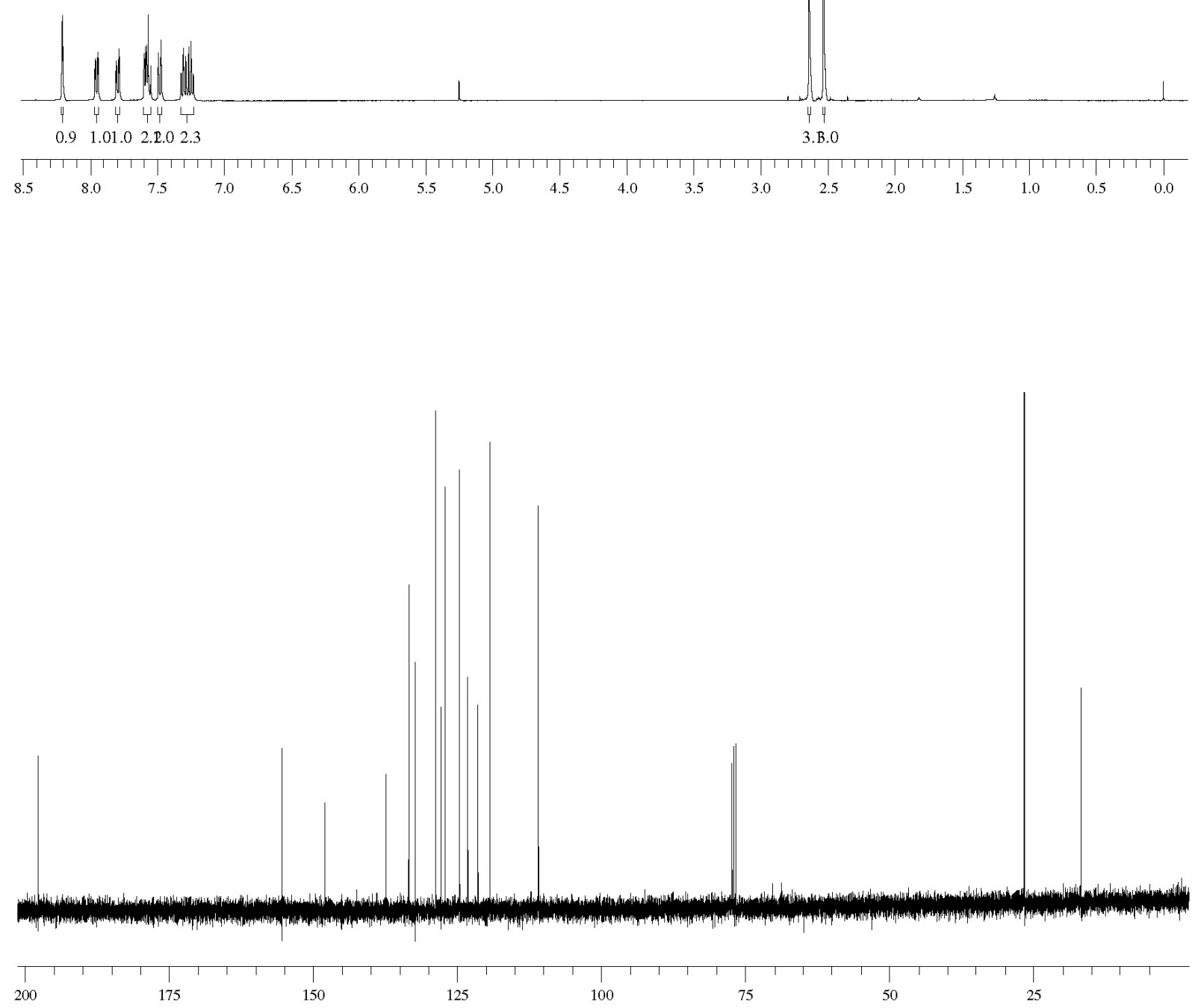

Figure S5. (up) ${ }^{1} \mathrm{H}$ NMR Spectrum (400 $\mathrm{MHz}, \mathrm{CDCl}_{3}$ ) of compound 3g; (down) ${ }^{13} \mathrm{C}$ NMR Spectrum $\left(100 \mathrm{MHz}, \mathrm{CDCl}_{3}\right.$ ) of compound $\mathbf{3 g}$. 


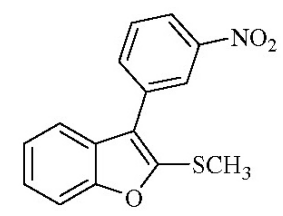

3h

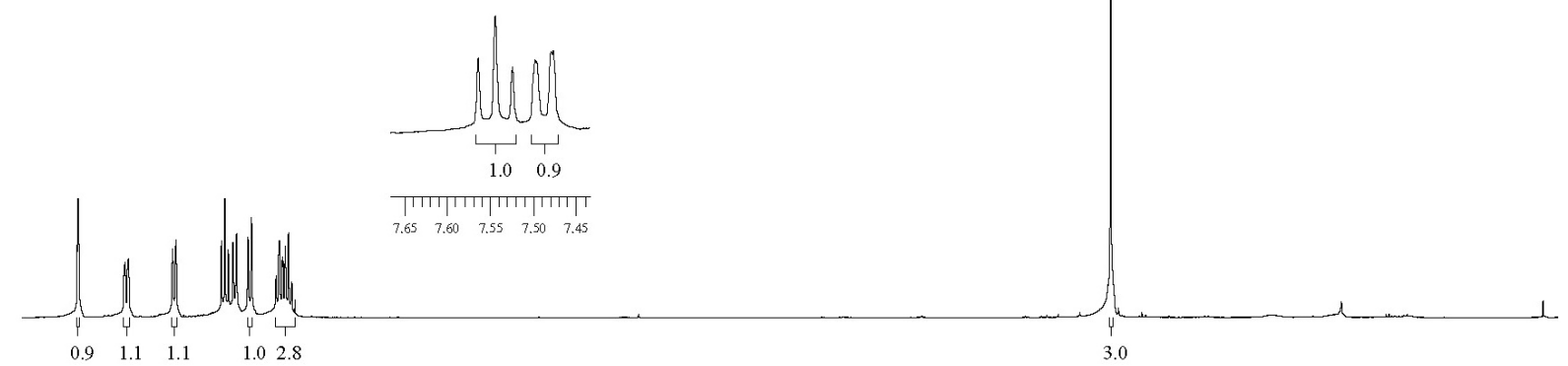

$\begin{array}{lllllll}0.9 & 1.1 & 1.1 & 1.0 & 2.8 & 3.0\end{array}$
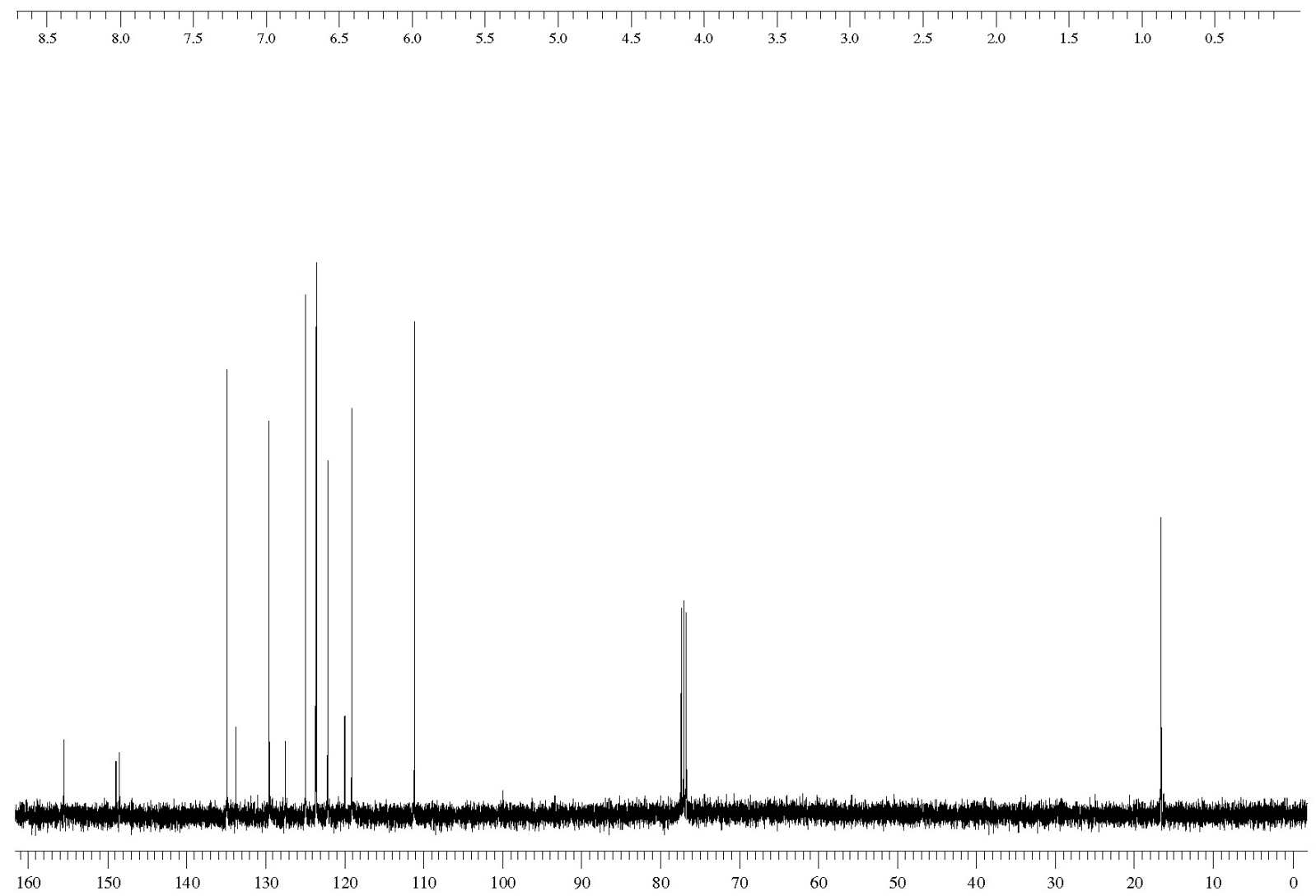

Figure S6. (up) ${ }^{1} \mathrm{H}$ NMR Spectrum (400 $\mathrm{MHz}, \mathrm{CDCl}_{3}$ ) of compound $\mathbf{3 h}$; (down) ${ }^{13} \mathrm{C} \mathrm{NMR} \mathrm{Spectrum} \mathrm{(100} \mathrm{MHz}, \mathrm{CDCl}_{3}$ ) of compound $\mathbf{3 h}$. 


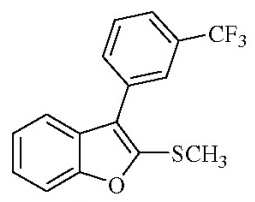

$3 \mathbf{i}$
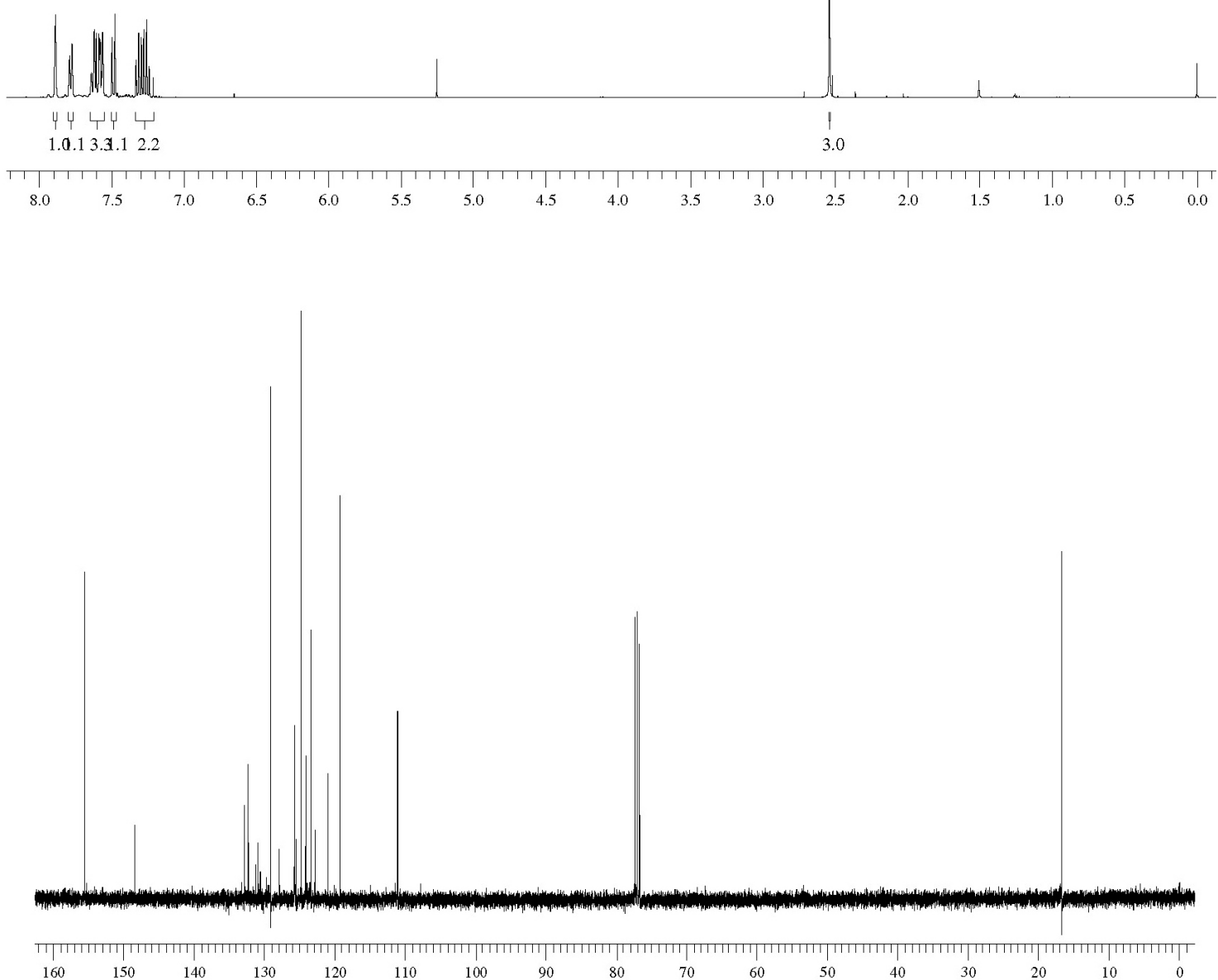

Figure S7. (up) ${ }^{1} \mathrm{H}$ NMR Spectrum (400 $\mathrm{MHz}, \mathrm{CDCl}_{3}$ ) of compound 3i; (down) ${ }^{13} \mathrm{C}$ NMR Spectrum $\left(100 \mathrm{MHz}, \mathrm{CDCl}_{3}\right.$ ) of compound $3 \mathbf{i}$. 

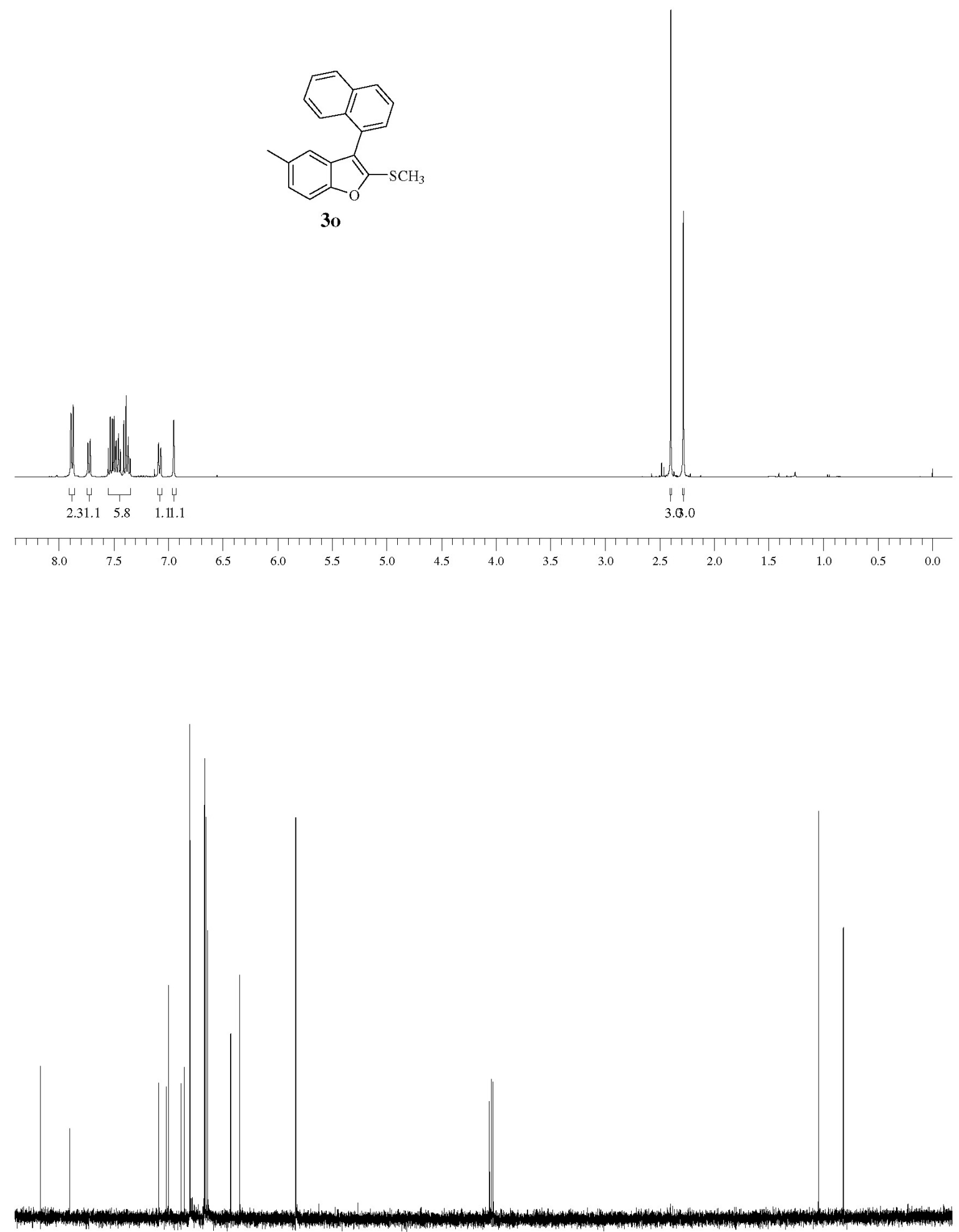

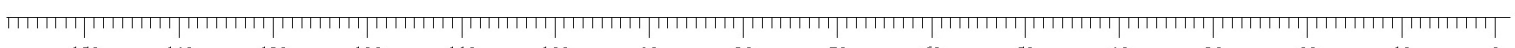
150
$120 \quad 110$
$100 \quad 90 \quad 80$
70
60
$\begin{array}{lllll}50 & 40 & 30 & 20 & 10\end{array}$

Figure S8. (up) ${ }^{1} \mathrm{H}$ NMR Spectrum (400 MHz, $\mathrm{CDCl}_{3}$ ) of compound 3o; (down) ${ }^{13} \mathrm{C}$ NMR Spectrum (100 MHz, $\mathrm{CDCl}_{3}$ ) of compound 3o. 

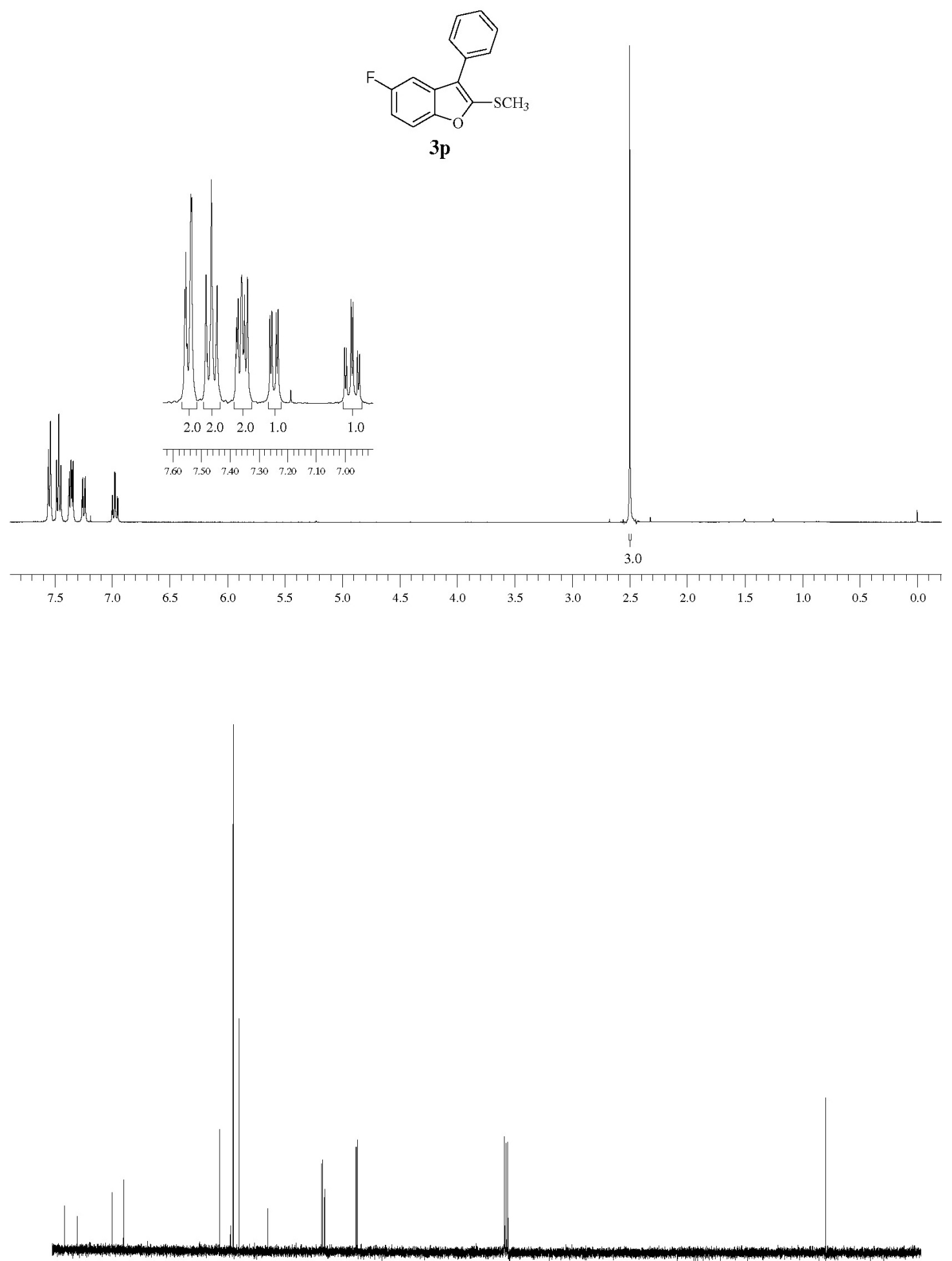

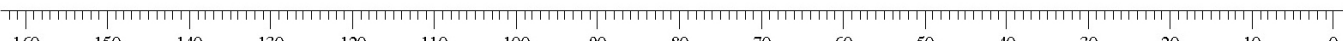

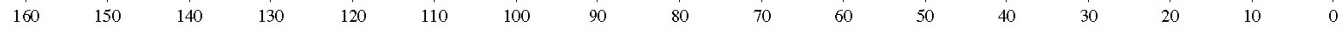

Figure S9. (up) ${ }^{1} \mathrm{H}$ NMR Spectrum (400 MHz, $\mathrm{CDCl}_{3}$ ) of compound 3p; (down) ${ }^{13} \mathrm{C}$ NMR Spectrum $\left(100 \mathrm{MHz}, \mathrm{CDCl}_{3}\right.$ ) of compound 3p. 
<smiles>Cc1ccccc1-c1c(C)oc2ccc(F)cc12</smiles>

$3 \mathbf{r}$
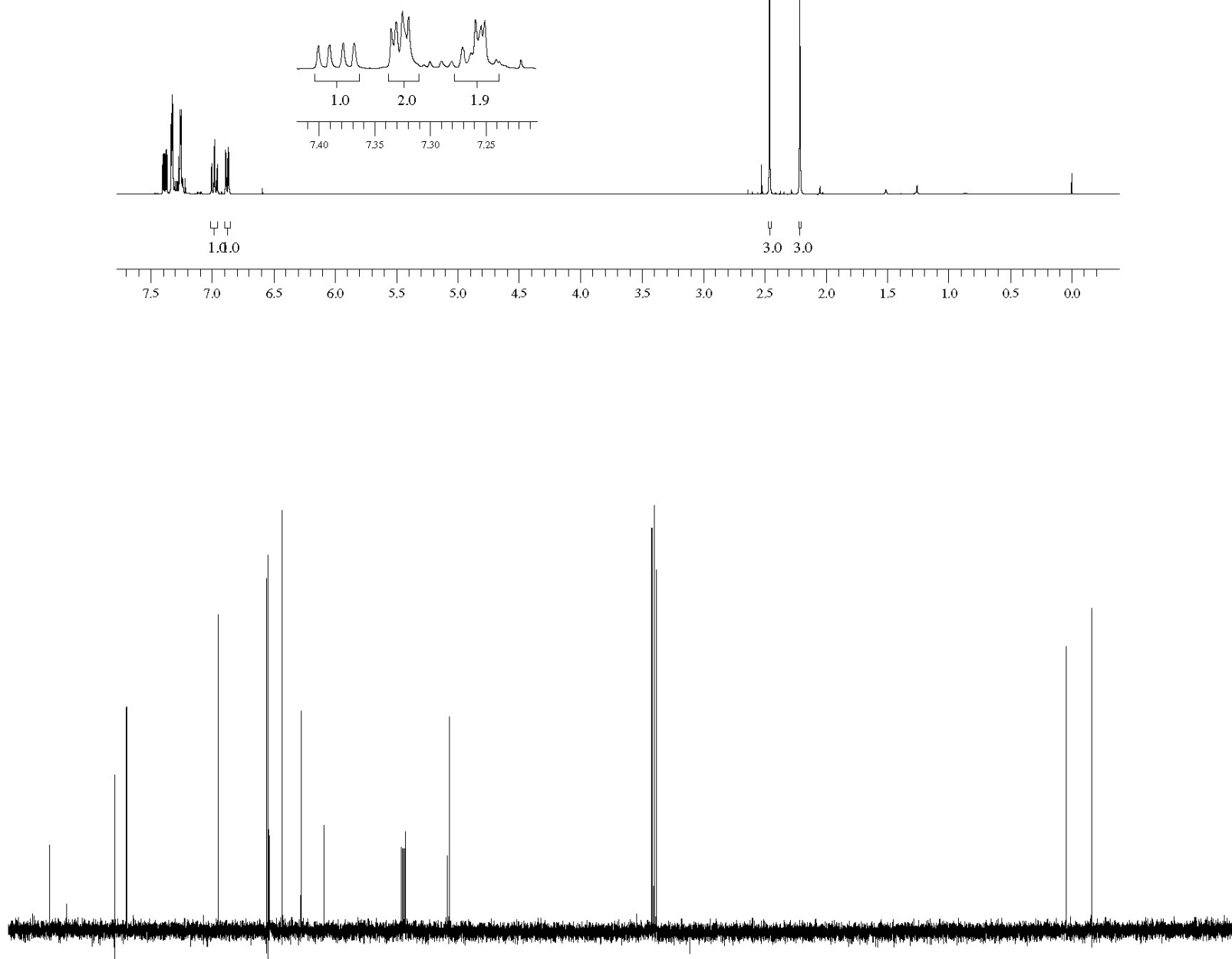

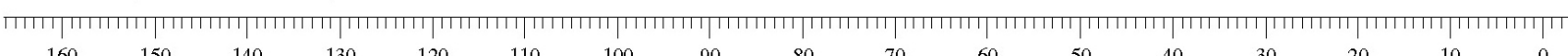

Figure S10. (up) ${ }^{1} \mathrm{H}$ NMR Spectrum (400 $\mathrm{MHz}, \mathrm{CDCl}_{3}$ ) of compound 3r; (down) ${ }^{13} \mathrm{C}$ NMR Spectrum $\left(100 \mathrm{MHz}, \mathrm{CDCl}_{3}\right.$ ) of compound 3r. 

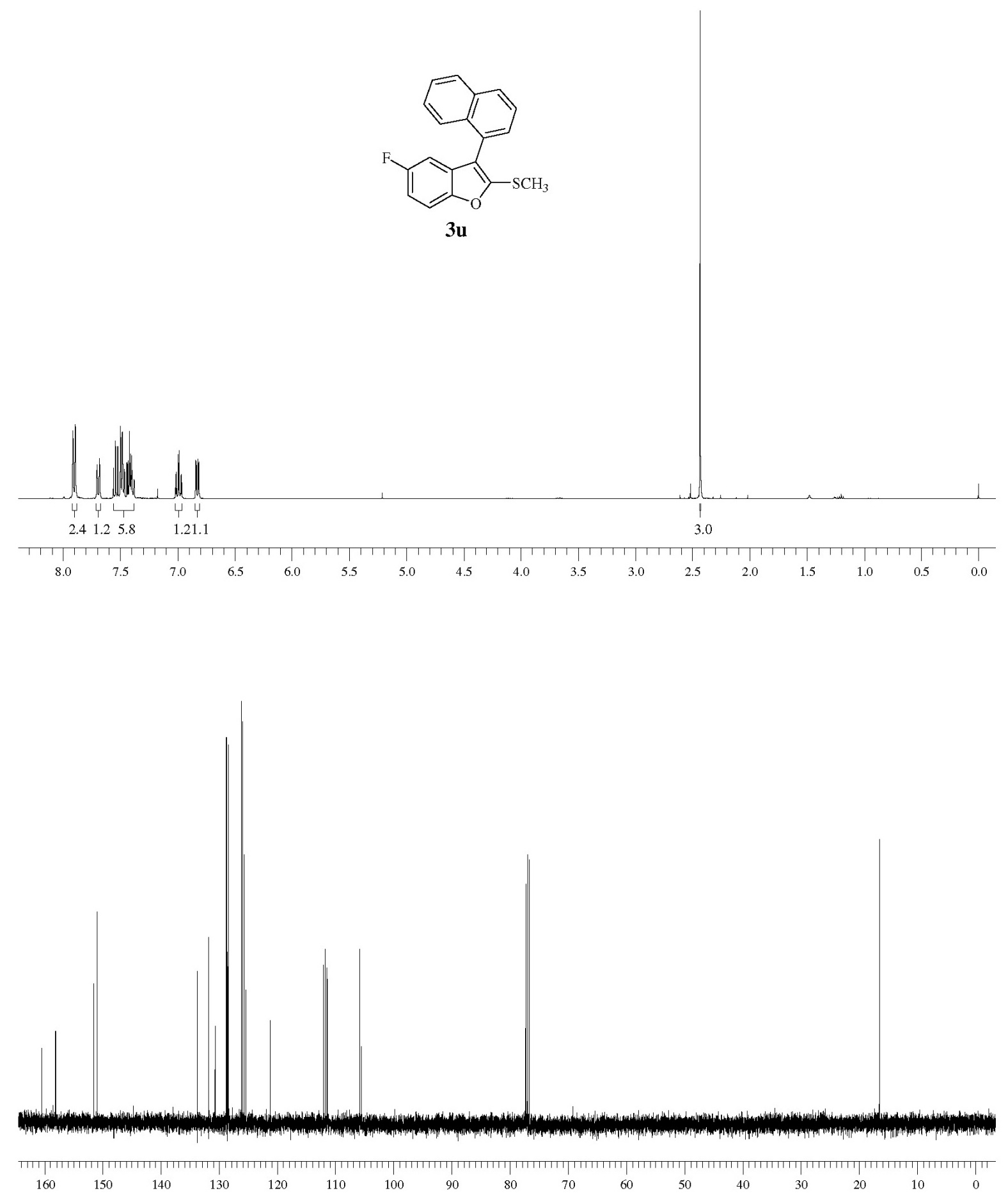

Figure S11. (up) ${ }^{1} \mathrm{H}$ NMR Spectrum (400 $\mathrm{MHz}, \mathrm{CDCl}_{3}$ ) of compound $\mathbf{3 u}$; (down) ${ }^{13} \mathrm{C}$ NMR Spectrum (100 MHz, $\mathrm{CDCl}_{3}$ ) of compound $\mathbf{3 u}$. 


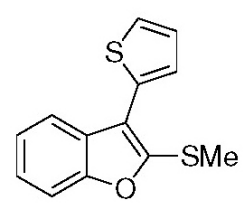

$3 \mathbf{x}$
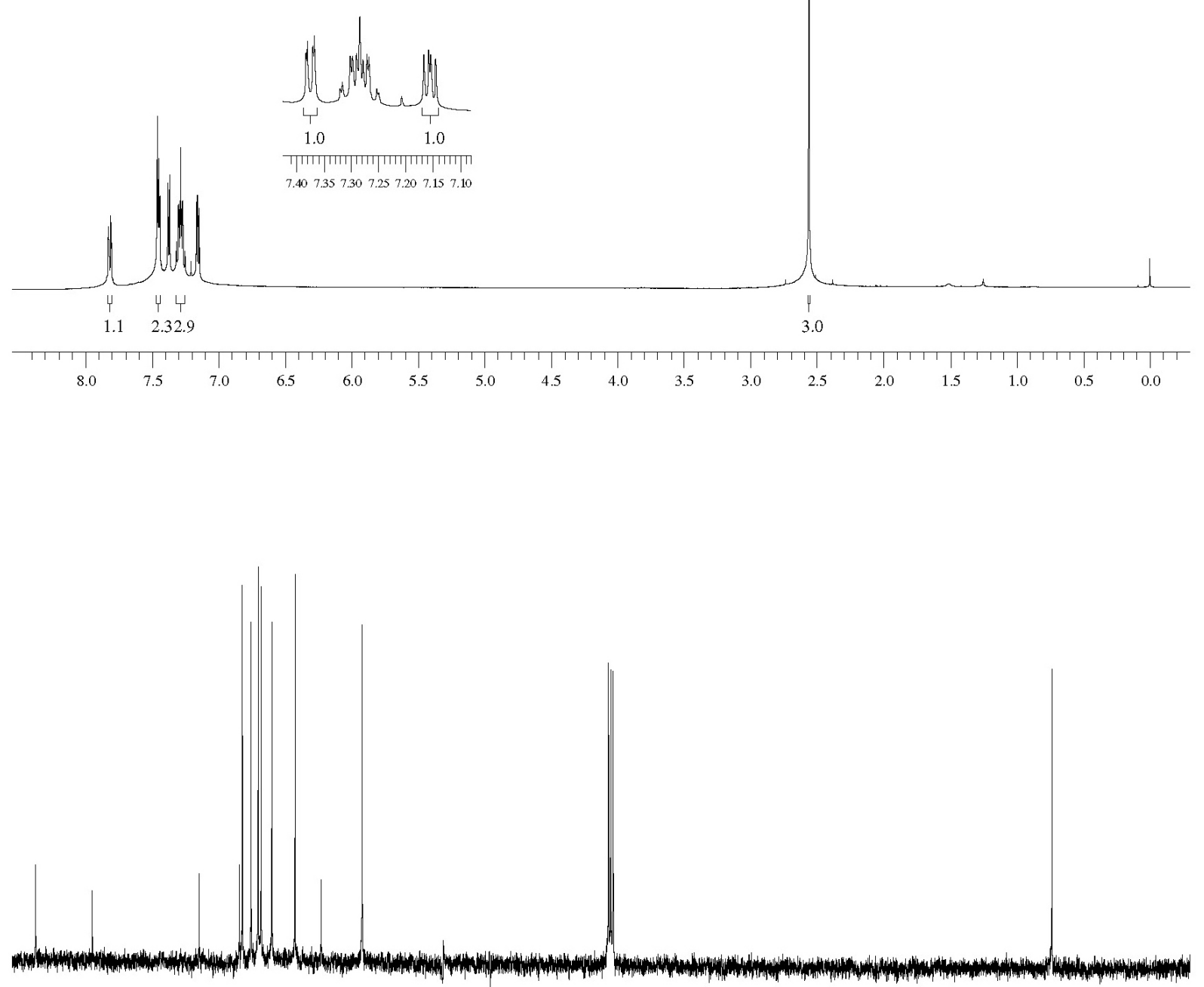

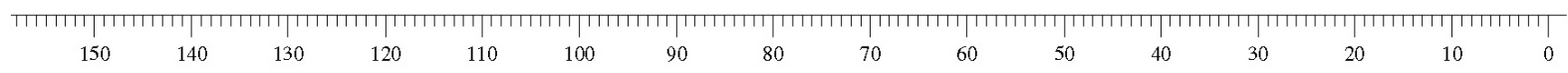

Figure S12. (up) ${ }^{1} \mathrm{H}$ NMR Spectrum (400 MHz, $\mathrm{CDCl}_{3}$ ) of compound $\mathbf{3 x}$; (down) ${ }^{13} \mathrm{C}$ NMR Spectrum (100 MHz, $\mathrm{CDCl}_{3}$ ) of compound $\mathbf{3 x}$. 